\title{
Thin, binary liquid droplets, containing polymer: an investigation of the parameters controlling film shape
}

\author{
A. D. Eales ${ }^{1}$, N. Dartnell ${ }^{2}$ \\ S. Goddard ${ }^{2}$ and A. F. Routh ${ }^{1} \dagger$ \\ ${ }^{1}$ Department of Chemical Engineering \& Biotechnology, University of Cambridge, Cambridge \\ CB2 3RA, UK \\ ${ }^{2}$ Cambridge Display Technology Ltd. (Company Number 02672530), Godmanchester PE29 \\ 2XG, UK
}

(Received ?; revised ?; accepted ?. - To be entered by editorial office)

For the fabrication of P-OLED displays, using inkjet printing, it is important to control the final shape resulting from evaporation of droplets containing polymer. Due to peripheral pinning and consequent outward capillary flow, a ring-like final shape is typically observed. This is often undesirable, with a spatially uniform film usually required. Several experimental studies have shown that binary liquid inks can prevent ring formation. There is no consensus of opinion on the mechanism behind this improvement. We have developed a model for the drying of thin, binary liquid droplets, based on thin-film lubrication theory and solve the governing equations to predict the final shape. White light interferometry experiments are conducted to verify the findings. In addition, we present the results of a linear stability analysis that identifies the onset of a surface tension differential driven instability. If the more volatile liquid is more abundant, an instability becomes increasingly likely.

Key words: Authors should not enter keywords on the manuscript, as these must be chosen by the author during the online submission process and will then be added during the typesetting process (see http://journals.cambridge.org/data/relatedlink/jfmkeywords.pdf for the full list)

\section{Introduction}

Evaporation of sessile droplets consisting of a solid component and a volatile liquid, is a topic with significant research interest. Understanding the film profile that results following complete evaporation is important in coating technologies, for example, paint or the distribution of active ingredients on plant leaves and/or weeds (Basi et al. (2013)). There are a significant number of biological applications, for example, deposit profiles can be used to identify diseases of the blood (Brutin et al. (2011)) and DNA microarrays (Heim et al. (2005); Angenendt (2005); Goldmann \& Gonzalez (2000); Deng et al. (2006); Dijskman \& Pierik (2008); Pierik et al. (2012)) can be used for disease diagnostics. The fabrication of electronic devices and circuits on the micro and nano scales (Sirringhaus et al. (2000); Kim et al. (2006); Sekitani et al. (2008); Ahn et al. (2009); Naqshbandi et al. (2012)) can be achieved through droplet evaporation. Other technologies that use 
inkjet printing, such as polymer-organic light-emitting diode displays (P-OLEDs), rely heavily on the dynamics of droplet drying (de Gans et al. (2004); de Gans \& Schubert (2004); van Dam \& Kuerten (2008); Dijskman et al. (2007)); this is the motivation of this research.

P-OLED displays boast several operational advantages over pre-existing technology, such as liquid crystal displays (LCDs), including improved viewing angles and the potential for curved and/or flexible screens. These stem from P-OLEDs being an emissive technology, in which light is emitted as a function of the electrical operation. There is no need for a backlight or a series of filters.

Such displays are manufactured by printing droplets containing the conducting polymer, followed by drying. Understanding the shape of the final light-emitting polymer profile is crucial for P-OLED applications. Specifically, we are interested in how the ink dispersion properties can be tailored to alter the film shape.

It is common to observe an undulating film profile, following complete evaporation of the volatile liquid. More material is deposited towards the droplets external boundaries than in the centre. This phenomenon is termed the coffee-ring effect (Deegan et al. (1997); Deegan (2000); Deegan et al. (2000); Gelderblom (2013)). It occurs when the contact line is pinned. Either surface roughness on the substrate or adsorption of the solid material can cause the pinning. The liquid evaporated towards the edge of the droplet, is replenished by an outward capillary flow that acts to ensure the droplet surface maintains an equilibrium shape. The outward capillary flow convects the solid towards the edge, where it is deposited as a ring. For P-OLED displays the current has a strong dependence on film thickness if the device is driven at a constant voltage. Thin areas may lead to hotspots as a result of the non-uniform thickness and emission profile.

Formation of coffee-rings has been the topic of considerable research, for reviews, refer to (Larson (2014)) and (Routh (2013)). It is sufficient here to say that there are broadly two methodologies for preventing coffee-ring formation. Either the pinning is prevented or the outward capillary flow is hindered. An example of the former is electrowetting (Eral et al. (2011)), in which the droplet boundaries are oscillated by the introduction of an alternating current. An example of the latter, is the introduction of a surfactant (Basi et al. (2013)), to cause an inward Marangoni flow counteracting the outward capillary flow. Other techniques include changing particle shape (Dugyala \& Basavaraj (2014); Vermant (2011); Yunker et al. (2011)) to increase the diffusion coefficient of the solid material, carefully choosing an electric field to control film shape (Wray et al. (2014)) and changing the $\mathrm{pH}$ to alter the Derjaguin Landau Verwey Overbeek (DLVO) interactions (Bhardwaj et al. (2010); Dugyala \& Basavaraj (2014)), to cause a strong attraction between the solid material and the substrate. None of these methods are ideal for POLED applications, where the droplet shape is set and additional additives are not permissible.

Another method, that could potentially be applied to mitigate coffee-ring formation, is the use of binary or multicomponent liquid inks (Kölpin et al. (2013); Bright et al. (2010); Wu et al. (2012)). This has the benefit that no additional material would need to be added to the final film, the improvement would be achieved by the ink formulation itself. The method has experimentally been shown to give flatter films, by many authors (Kölpin et al. (2013); Tekin et al. (2004); de Gans \& Schubert (2004); Park \& Moon (2006); Teichler et al. (2013)), for example, Teichler et al. (Teichler et al. (2013)) have shown that a large difference in volatilities improves film shape. However, the majority of research has been conducted for thick droplets. It has been postulated that the mechanism driving this improvement is a solvent induced Marangoni circulation (de Gans \& Schubert (2004); Park \& Moon (2006); Yamaue et al. (2006)). A liquid composition gradient can exist 
within the droplet and hence a surface tension gradient will exist along the droplet. From consideration of the Young-Laplace equation $(p=-\gamma \kappa$, where $p$ is the fluid pressure, $\gamma$ is the surface tension and $\kappa$ is the curvature), one might expect a surface flow from low to high surface tension areas, when the curvature of the droplet is positive. The circulation mechanism is however not relevant for our application, in which the droplets are thin enough that there are no depthwise gradients in composition. Many authors report that an improvement in film shape is gained if the more volatile liquid has a higher surface tension (Kim et al. (2006); Park \& Moon (2006); Talbot et al. (2012); Yang et al. (2012); Chow et al. (2009)), however, some authors have observed the opposite (de Gans \& Schubert (2004); Karabasheva et al. (2006); Yamaue et al. (2006)). This suggests that the solvent induced Marangoni circulation is not always the dominant mechanism. As pointed out by Kaneda et al. (Kaneda et al. (2008)), the direction of Marangoni circulation could depend on whether or not the droplet is pinned. Babatunde et al. (2013) suggests the reason for the improvement in shape in many of the given examples could be due to depinning, rather than Marangoni circulation. It has also been observed that solute induced Marangoni circulation can influence the final deposit shape but this cannot alone account for the removal of coffee-ring formation (Babatunde et al. (2013)). With the exception of Yamaue et al. (Yamaue et al. (2006)), we are not aware of any other attempts to model the evaporation dynamics and resulting final film profile for binary liquid droplets. Yamaue et al. (Yamaue et al. (2006)) consider a thick droplet of anisole / ethyl acetate and extend the $\mathrm{Hu}$ and Larson model (Hu \& Larson (2002, $2005 a, b, 2006))$ to predict the optimal mixing ratio of solvent.

As noted by previous authors (Babatunde et al. (2013)), there is a need to model the evaporation of binary liquid droplets. Flow instabilities can form in binary liquid droplets, as mentioned by Sefiane et al. (Sefiane et al. (2008)) and Kaneda et al. (Kaneda et al. (2008)), amongst others. This possibility adds a level of complexity to the work required. In this paper, we build on previous thin-film lubrication models (Routh \& Russel (1998); Salamanca et al. (2001); Ozawa et al. (2005); Tarasevich et al. (2013); Wray et al. (2014); Eales et al. $(2015 a, b))$ and extend the analysis to ideal, binary liquids containing polymer.

During drying, solids fraction increases due to evaporation of the volatile liquid. When the solids content is sufficiently large, consolidated regions can form. In the case of polymer, a gel typically forms. The position of the liquid/gel front that results, is tracked continuously. In order to determine whether the numerical solutions make sense, we perform white light interferometry experiments on the residues of inkjetted droplets.

With regard to the evaporative flux distribution across the surface of a droplet, much debate remains. It was shown by Fischer (Fischer (2002)) that the evaporative flux distribution can alter internal flows and in turn the final film profile. For small, pure liquid droplets, with stagnant surroundings, an enhancement in the evporation rate towards the edge of the droplets is expected. This occurs because of the mass transfer limitation being diffusion of vapour away from the droplet surface. This is not the case for large, pure liquid droplets, in a well ventilated atmosphere, where a spatially uniform evaporative flux profile is expected. Through digital holographic interferometry experiments, it has been shown that the profile differs markedly from vapour diffusion models (Dahaeck et al. (2014)). To our knowledge this is the first attempt at modeling the evaporation dynamics of a binary liquid droplet containing polymer, in the thin film, lubrication regime. For that reason, we wish to keep the model as simple as possible, to delineate the important mechanisms pertinent to selection of liquids. The evaporative flux distribution is taken as spatially uniform for each individual liquid, with any spatial variation resulting solely from liquid compositional variations. The present model could be extended, to include spatially varying evaporation, if necessary. 
Since there is the possibility of an instability occurring in the droplet height, within the thin droplets, we use a linear stability analysis to predict its onset. This analysis has previously been used for thin films, for example, by Yeo et al. (Yeo et al. (2003)), in which the stability of a flat film on a heated substrate is predicted. Our work considers the stability of a binary liquid droplet. The geometry can result in a significant liquid compositional gradient within the droplet. The presence of polymer and diffusion are ignored for this analysis, since they are both likely to have a stabilising effect.

The aim of this paper is to discover the important parameters controlling the final film shape for inkjetted binary liquid droplets, containing light-emitting polymer. We hope that this research will provide an insight into selection of the liquid components in order to mitigate coffee-ring formation.

\section{Mathematical model}

Consider a droplet consisting of a suspension of a single, solid material (e.g. polymer), in a perfectly miscible, binary mixture of volatile liquid components. Component 1 is defined as the more volatile liquid (volumetric evaporation rate per unit area $E_{1}{ }^{\star}$, density $\rho_{1}^{\star}$, viscosity $\mu_{1}^{\star}$, surface tension $\left.\gamma_{1}^{\star}\right)$ and component 2 as the less volatile liquid (volumetric evaporation rate per unit area $E_{2}{ }^{\star}$, density $\rho_{2}{ }^{\star}$, viscosity $\mu_{2}{ }^{\star}$, surface tension $\gamma_{2}{ }^{\star}$. Properties marked with an ${ }^{\star}$, are for the pure liquid. The solid material is initially uniformly distributed with volume fraction $\phi_{0}$.

\subsection{Assumptions $\& 3$ mixture properties}

For the binary liquid, we consider an ideal mixture. Non-ideal, partially miscible systems are not treated in this work, but it is possible to extend the theory if necessary. In addition, it is assumed that the surrounding atmosphere is an ideal gas. These assumptions enable the use of Raoult's law, such that the vapour pressure of a liquid component is the product of that components' vapour pressure, when pure and its' liquid phase mole fraction. The total vapour pressure of the liquid phase, $p_{v}$, is hence

$$
p_{v}=p_{1}{ }^{\star} x_{1}+p_{2}{ }^{\star}\left(1-x_{1}\right)
$$

Where $x_{1}$ is the liquid phase mole fraction of component 1 , on a solid free basis and $p_{1}{ }^{\star}$ and $p_{2}{ }^{\star}$ are the vapour pressures of each, pure, liquid.

Assuming that the concentration of the volatile components in the surrounding atmosphere are zero and the system is well ventilated, the pure liquid, evaporation rates of each component are given by

$$
\begin{aligned}
& E_{1}{ }^{\star}=k_{1}{ }^{\star} p_{1}{ }^{\star} \\
& E_{2}{ }^{\star}=k_{2}{ }^{\star} p_{2}{ }^{\star}
\end{aligned}
$$

Where $k_{i}{ }^{\star}$ is the mass transfer coefficient for the transfer of pure liquid, $i$, to the vapour phase.

By applying Raoult's law, the total liquid evaporation rate, $E$, is

$$
E=E_{1}{ }^{\star} x_{1}+E_{2}{ }^{\star}\left(1-x_{1}\right)
$$

For the mixture viscosity, a simple linear relation is adopted between the viscosities of the two liquids. It should be noted that the viscosity does increase with solids content, however, as we will discuss later, the flow within the droplet is dominated by surface 


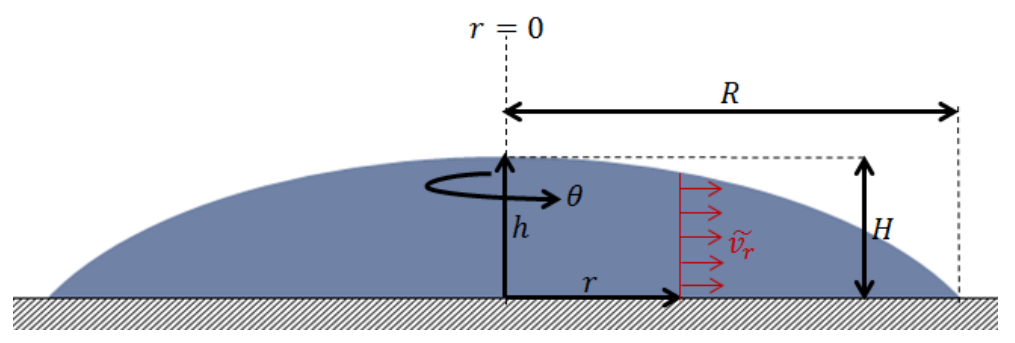

FiguRE 1. Sketch of a droplet residing on a flat substrate.

tension effects. Later, we will show that the nature of this expression is unimportant, since surface tension effects dominate the flow profile.

$$
\mu=\mu_{1}^{\star} x_{1}+\mu_{2}^{\star}\left(1-x_{1}\right)
$$

To fit the surface tension of the binary liquid mixture as a function of liquid composition, one might crudely consider a linear relation. In order for the surface energy of the droplet to be minimised, however, there is a preferential partition of the lower surface tension liquid to the droplet surface (Schmidt et al. (1966); Escobedo \& Ali Mansoori (1998); Hildebrand \& Scott (1964); Suri \& Ramakrishna (1968)). For this reason and following Hildebrand and Scott (Hildebrand \& Scott (1964)) and Suri and Ramakrishna (Suri \& Ramakrishna (1968)), the surface tension is fitted using

$$
\gamma=\gamma_{1}{ }^{\star} x_{1}+\gamma_{2}{ }^{\star}\left(1-x_{1}\right) \pm \frac{A}{2 \cdot R_{g} \cdot T}\left(\gamma_{1}^{\star}-\gamma_{2}^{\star}\right) x_{1}\left(1-x_{1}\right)
$$

Where $A$ is the surface occupancy of the molecules $\left(\mathrm{cm}^{2} \mathrm{~mol}^{-1}\right)$ and is approximately the same for each component if the mixture is ideal, $R_{g}$ is the universal gas constant and $T$ is the temperature, in Kelvin. The \pm term is used to ensure that the surface tension is always reduced compared to the linear relation. Note that the expression above does not include any solids impact on surface tension. By performing experiments using inks containing different concentrations of light-emitting polymer, we did not observe a notable influence on surface tension.

\subsection{Scaling terms and simplifications}

Due to either surface inhomogeneities or adsorption of the solid material to the substrate, the three phase contact-line is assumed to be pinned. The model considers an axisymmetric droplet, in a cylindrical coordinate system (radius $r$, height $h$, azimuth $\theta$ ), such that velocity and gradients in the azimuthal direction are negligible $\left(v_{\theta}=0, \frac{\partial}{\partial \theta}=0\right)$. As can be see in figure 1 , the horizontal length scale is the droplet radius, $R$ and the vertical length scale is the initial droplet height, $H$. An extension to droplets with a non-circular cross-section, as in van Dam \& Kuerten (van Dam \& Kuerten (2008)), could be an interesting topic for future work on P-OLED displays.

Scaling is arbitrarily performed on the basis of the less volatile liquid, as outlined in table 1.

Typically, $R$ is about $125 \mu \mathrm{m}$. The Bond number, Bo, can be used to compare the relative magnitudes of gravity and surface tension on the droplet. It is found that typical values for the Bond number are very small and consequently surface tension dominates at this length scale. Gravitational sagging of the droplet interface can hence be neglected. 


Property
air-liquid interface height, $h$
vertical height, $z$
horizontal position, $r$
vertical velocity, $v_{z}$
horizontal velocity, $v_{r}$
viscosity, $\mu$
surface tension, $\gamma$
density, $\rho$
pressure, $p$
time, $t$

Scaling term
$H$
$H$
$R$
$E_{2}^{\star}$
$\frac{R \cdot E_{2}{ }^{\star}}{H}$
$\mu_{2}{ }^{\star}$
$\gamma_{2}{ }^{\star}$
$\rho_{2}^{\star}$
$\frac{\mu_{2}{ }^{\star} \cdot R^{2} \cdot E_{2}}{H^{3}}$
$\frac{H}{E_{2}{ }^{\star}}$

TABLE 1. Scaling terms used to cast non-dimensional properties. A * denotes saturated parameter values.

$$
B o=\frac{\rho_{2}{ }^{\star} g R^{2}}{\gamma_{2}^{\star}} \sim 10^{-3}
$$

Where $g$ is the acceleration due to gravity. The liquid density $\left(\rho_{2}{ }^{\star}=1084 \mathrm{~kg} \mathrm{~m}^{-3}\right)$ (Garcia et al. (1991)) and air-liquid surface tension $\left(\gamma_{2}{ }^{\star}=3.8 \times 10^{-2} \mathrm{~N} \mathrm{~m}^{-1}\right)$ (Wohlfarth (2008)) are for methyl benzoate at 1 atmosphere and $20^{\circ} \mathrm{C}$.

The Reynolds number, $R e$, is very small. This enables simplification of the governing equations, by the creeping flow assumption, in which inertial effects are neglected. Furthermore, we consider thin films, for which the lubrication approximation $\left(R e \cdot \frac{H^{2}}{R^{2}} \ll 1\right)$ holds.

$$
R e=\frac{\rho_{2}{ }^{\star} E_{2}{ }^{\star} R}{\mu_{2}{ }^{\star}} \sim 10^{-7}
$$

The evaporative flux per unit area $\left(E_{2}{ }^{\star}=2.0 \times 10^{-9} \mathrm{~m}^{3} \mathrm{~m}^{-2} \mathrm{~s}^{-1}\right)$ was measured by the mass loss from a petri dish containing pure methyl benzoate, at $20^{\circ} \mathrm{C}$. The viscosity $\left(\mu_{2}{ }^{\star}=1.8 \times 10^{-3} \mathrm{~N} \mathrm{~s} \mathrm{~m}^{-2}\right.$ ) (Garcia et al. (1991)) is again for methyl benzoate at 1 atmosphere and $20^{\circ} \mathrm{C}$..

The strength of diffusion in the horizontal direction will be a function, not only of particle size but also shape. We account for the relative strength of diffusion by using the Péclet number, $P e_{p}$, which is the ratio of the rate of convection to diffusion. Generally the diffusion is weak. As solids content increases, the viscosity increases. This results in a smaller diffusion coefficient and a larger Péclet number. In our numerical solutions we will use the base case $P e_{p} \rightarrow \infty$, but we will handle a few cases of finite $P e_{p}$ to investigate weak diffusion.

$$
P e_{p}=\frac{R^{2} E_{2}^{\star}}{H D_{p}} \approx 3
$$

$D_{p}$ is the Stokes-Einstein diffusion coefficient for the solid material, in the liquid medium $\left(1.2 \times 10^{-12} \mathrm{~m}^{2} \mathrm{~s}^{-1}\right.$ for a dilute suspension of $100 \mathrm{~nm}$, rigid spheres $)$ and a typical value for $H$ is $\sim 10 \mu \mathrm{m}$. We treat the solid as a suspension of non-interacting spheres. Note that for polymer chains the validity of the rigid sphere assumption depends on the quality of the solvent. This could provide a topic for future research.

For the liquid-liquid diffusion, we use the liquid-liquid Péclet number, $P e_{l l}$. 


$$
P e_{l l}=\frac{R^{2} E_{2}^{\star}}{H D_{l l}} \sim 10^{-3}
$$

The diffusion coefficient of the 'solute' liquid, 1 , through the 'solvent' liquid, 2 , is $D_{l l}$. A typical order of magnitude for liquids is $10^{-9} \mathrm{~m}^{2} \mathrm{~s}^{-1}$. For the numerical solutions we initially examine the case without diffusion $\left(P e_{l l} \rightarrow \infty\right)$, to understand what would be expected to happen if there were no liquid-liquid diffusion. We then move on to consider finite $P e_{l l}$. Section 4.2 is designated to the treatment of liquid-liquid diffusion and its significance.

The relative importance of viscous to surface tension forces is governed by the magnitude of the Capillary number, $\mathrm{Ca}$. In the derivation of the governing equations, which follows in section 2.3, it is shown that the Capillary number is:

$$
C a=\frac{3 \mu_{2}{ }^{\star} R^{4} E_{2}{ }^{\star}}{\gamma_{2}{ }^{\star} H^{4}} \sim 10^{-5}
$$

It is found under most conditions that surface tension effects dominate viscous ones. Even with an increase in viscosity of several orders of magnitude, due to increased solids content, the viscosity has no impact on film shape. Hence, all numerical solutions provided in this work will consider the small $\mathrm{Ca}$ limit. For the simulations, the small capillary number limit is taken to be $C a=10^{-3}$; this ensures that surface tension dominates whilst achieving an acceptable simulation time. Previous works (Fischer (2002)) and (Eales et al. $(2015 a)$ ) have shown that if the Capillary number could be made large, coffee-ring formation would be avoided.

As the volatile liquid mixture evaporates, the volume fraction of the solid component, $\phi$, increases from an initial value of typically $1 \%$ or less. Due to packing or gelling, a maximum value, $\phi_{\max }$, will eventually be reached. It is assumed that $\phi_{\max }$ is the same for both liquids. The viscosity of the dispersion will increase with increasing solid volume fraction. The expression we use to account for this, following Krieger and Dougherty (Krieger \& Dougherty (1959)), is shown in equation 2.12.

$$
\frac{\mu}{\mu_{0}}=\left[1-\frac{\phi}{\phi_{\max }}\right]^{-n}
$$

Note that if the if polymer solutions are considered, a more specific, alternative relationship would be required. However, due to the very small capillary number, this viscosity correction term generally has no impact on the final film shape.

It is assumed that the presence of the solid material does not alter the surface tension significantly. Thus, the solutal Marangoni effect is neglected but it is possible to extend this work to account for it, if required.

It has been argued in previous works (Ozawa et al. (2005); Tarasevich et al. (2013)), that when the volume fraction reaches $\phi_{\max }$, evaporation will be completely suppressed. If this were the case, we would expect the final deposit to be wet. In other words, a fraction $\left(1-\phi_{\max }\right)$ of the final film volume would still contain liquid. In practice this is not observed, meaning that evaporation, whilst significantly hindered, cannot be completely suppressed. This is accounted for by using a hindrance fraction, $f_{h}$, such that the evaporation in close-packed/gelled regions is hindered in the following manner

In regions where $\phi=\phi_{\max }: \quad E_{h}(r)=\left(1-f_{h}\right) E(r)$, where $0 \leqslant f_{h}<1$

$E_{h}$ is the evaporation rate in the close-packed/gelled region, $E$ is the evaporation rate 
if there were no hindrance to evaporation. In the base case, the hindrance fraction, $f_{h}$, is chosen to be constant at 0.95 .

Since the purpose of this work is to predict trends and provide guidance in selection of binary liquid inks for inkjet printing applications, we will not concern ourselves greatly with the subtleties of edge enhanced evaporation profiles. $E_{1}{ }^{\star} \& E_{2}{ }^{\star}$ are assumed to be spatially uniform. The evaporation rate, $E$ is taken to be a function of position and time, solely because $x_{1}$ is a function of position.

$$
E(r)=E_{1}{ }^{\star} x_{1}(r)+E_{2}^{\star}\left(1-x_{1}(r)\right)
$$

\subsection{Derivation of the governing equations}

For pure liquid droplets, the governing equations for droplet height and volume fraction of solid material have been derived by Routh \& Russel (1998), Salamanca et al. (2001), Ozawa et al. (2005), Tarasevich et al. (2013), Wray et al. (2014), Eales et al. (2015a), van Dam \& Kuerten (2008), Eales et al. (2015b) and Eales (2015). Here, we extend the work to binary liquid mixtures. In addition, we derive the governing equation for the liquid composition and allow for evaporation hindrance upon gelation.

Starting with the incompressible, axisymmetric, steady state, Navier-Stokes equations, the non-dimensionalisation can be performed using the scalings outlined in table 1. Applying the lubrication assumption, we find that for the thin film there is negligible depthwise gradient in pressure. The horizontal velocity and the surface pressure gradient are related by

$$
\frac{d \bar{p}}{d \bar{r}}=\bar{\mu} \frac{\partial^{2} \overline{v_{r}}}{\partial \bar{z}^{2}}
$$

Where $\bar{\mu}$ is the non-dimensional viscosity, neglecting the influence of polymer, given by

$$
\bar{\mu}=x_{1} \frac{\mu_{1}^{\star}}{\mu_{2}^{\star}}+\left(1-x_{1}\right)
$$

By integrating 2.15 twice, with respect to the vertical coordinate, an expression for the horizontal velocity can be determined. The no-slip boundary condition is used at the substrate $\left(\bar{z}=0, \bar{v}_{r}=0\right)$ and the tangential stress at the droplet surface must be equal to the surface tension gradient, following equation 2.17. Note that the non-linear term in equation 2.6 is neglected in this balance. This is because an order of magnitude analysis conducted using a typical value for the surface occupancy of melecules, A, from Suri \& Ramakrishna (1968), shows that the non-linear contribution is of negligble magnitude.

$$
\begin{gathered}
\left.\bar{\mu} \frac{\partial \overline{v_{r}}}{\partial \bar{z}}\right|_{\bar{z}=\bar{h}}=\left.\frac{\gamma_{2}{ }^{\star} H^{2}}{\mu_{2}{ }^{2} R^{2} E_{2}}\left(\frac{\gamma_{1}^{\star}}{\gamma_{2}{ }^{\star}}-1\right) \frac{\partial x_{1}}{\partial \bar{r}}\right|_{\bar{z}=\bar{h}} \\
\bar{v}_{r}=\left(\frac{\bar{z}^{2}-2 \bar{h} \bar{z}}{2 \bar{\mu}}\right) \frac{d \bar{p}}{d \bar{r}}+\left.\frac{1}{\bar{\mu}} \frac{R^{2}}{H^{2}}\left(\frac{\gamma_{1}^{\star}}{\gamma_{2}{ }^{\star}}-1\right) \bar{z} \frac{\partial x_{1}}{\partial \bar{r}}\right|_{\bar{z}=\bar{h}}
\end{gathered}
$$

The pressure gradient is found by differentiating the Young-Laplace equation ( $p=$ $-\gamma . \kappa)$, where $\kappa$ is the mean curvature of the droplet surface. A standard expression for $\kappa$ can be derived using differential geometry. Within the lubrication approximation, the pressure is 


$$
\bar{p}=-\frac{\gamma_{2}{ }^{\star} H^{4}}{\mu_{2}{ }^{\star} R^{4} E_{2}} \bar{\gamma}\left(\frac{\partial^{2} \bar{h}}{\partial \bar{r}^{2}}+\frac{1}{\bar{r}} \frac{\partial \bar{h}}{\partial \bar{r}}\right)
$$

Where $\bar{\gamma}$ is the non-dimensional surface tension at the air-liquid interface, given by

$$
\bar{\gamma}=\frac{\gamma_{1}^{\star}}{\gamma_{2}^{\star}} x_{1}+\left(1-x_{1}\right) \pm \frac{A}{2 R T}\left(\frac{\gamma_{1}^{\star}}{\gamma_{2}^{\star}}-1\right) x_{1}\left(1-x_{1}\right)
$$

Subsequent differentiation yields the pressure gradient as

$$
\frac{d \bar{p}}{d \bar{r}}=-\frac{\gamma_{2}{ }^{\star} H^{4}}{\mu_{2}{ }^{\star} R^{4} E_{2}{ }^{\star}} \frac{\partial}{\partial \bar{r}}\left[\bar{\gamma}\left(\frac{\partial^{2} \bar{h}}{\partial \bar{r}^{2}}+\frac{1}{\bar{r}} \frac{\partial \bar{h}}{\partial \bar{r}}\right)\right]
$$

Hence, using equation 2.18 , the horizontal velocity is

$$
\bar{v}_{r}=\frac{\gamma_{2}{ }^{\star} H^{4}}{2 \mu_{2}{ }^{\star} R^{4} E_{2}{ }^{\star}} \frac{1}{\bar{\mu}}\left[\left(2 \bar{h} \bar{z}-\bar{z}^{2}\right) \frac{\partial}{\partial \bar{r}}\left[\bar{\gamma}\left(\frac{\partial^{2} \bar{h}}{\partial \bar{r}^{2}}+\frac{1}{\bar{r}} \frac{\partial \bar{h}}{\partial \bar{r}}\right)\right]+\left.2 \frac{R^{2}}{H^{2}}\left(\frac{\gamma_{1}^{\star}}{\gamma_{2}^{\star}}-1\right) \bar{z} \frac{\partial x_{1}}{\partial \bar{r}}\right|_{\bar{z}=\bar{h}}\right]
$$

In the lubrication regime, we are interested in the horizontal flow within the droplet and ignore depthwise gradients. It is convenient to consider the vertically averaged horizontal velocity.

$$
\begin{gathered}
\tilde{\overline{v_{r}}}=\frac{1}{\bar{h}} \int_{\bar{z}=0}^{\bar{z}=\bar{h}} \overline{v_{r}} d \bar{r}=\frac{\bar{h}^{2} \frac{\partial}{\partial \bar{r}}\left[\bar{\gamma}\left(\frac{\partial^{2} \bar{h}}{\partial \bar{r}^{2}}+\frac{1}{\bar{r}} \frac{\partial \bar{h}}{\partial \bar{r}}\right)\right]+\left.\frac{3 R^{2}}{2 H^{2}}\left(\frac{\gamma_{1}{ }^{\star}}{\gamma_{2}{ }^{\star}}-1\right) \bar{h} \frac{\partial x_{1}}{\partial \bar{r}}\right|_{\bar{z}=\bar{h}}}{C a \bar{\mu}} \\
\text { where } C a=\frac{3 \mu_{2}{ }^{\star} R^{4} E_{2}^{\star}}{\gamma_{2}{ }^{\star} H^{4}}
\end{gathered}
$$

By examining the boundary condition at the droplet surface, defined in equation 2.17, two limits for the vertically averaged horizontal velocity can be considered, as follows

Velocity Limit 1: $\bar{\mu}$ and $\left.\frac{\partial \bar{v}_{r}}{\partial \bar{z}}\right|_{\bar{z}=\bar{h}}$ are order unity

In this limit, the air/liquid interface approximately has zero-shear stress. This is because a strong surface flow rapidly alters the surface composition to ensure there is no gradient in surface tension. This is a localised effect and the liquid composition can still vary in the bulk liquid, below the surface.

$$
\begin{gathered}
\left.\frac{\partial x_{1}}{\partial \bar{r}}\right|_{\bar{z}=\bar{h}} \sim \frac{\mu_{2}{ }^{\star} R^{2} E_{2}^{\star}}{\gamma_{2}{ }^{\star} H^{2}} \approx 10^{-8} \\
\tilde{\overline{v_{r}}} \approx \frac{\bar{h}^{2} \frac{\partial}{\partial \bar{r}}\left[\bar{\gamma}\left(\frac{\partial^{2} \bar{h}}{\partial \bar{r}^{2}}+\frac{1}{\bar{r}} \frac{\partial \bar{h}}{\partial \bar{r}}\right)\right]}{C a \bar{\mu}}
\end{gathered}
$$

Velocity Limit 2: $\left.\frac{\partial x_{1}}{\partial \bar{r}}\right|_{\bar{z}=\bar{h}}$ is order unity

In this limit, the vertical gradient in horizontal velocity is very large at the air/liquid interface. The curvature induced, capillary flow is negligible compared to the Marangoni flow. 


$$
\begin{gathered}
\left.\frac{\partial \overline{v_{r}}}{\partial \bar{z}}\right|_{\bar{z}=\bar{h}} \sim \frac{\gamma_{2}{ }^{\star} H^{2}}{\mu_{2}{ }^{\star} R^{2} E_{2}{ }^{\star}} \approx 10^{8} \\
\tilde{\overline{v_{r}}} \approx \frac{\left.\frac{3 R^{2}}{2 H^{2}}\left(\frac{\gamma_{1}^{\star}}{\gamma_{2}{ }^{\star}}-1\right) \bar{h} \frac{\partial x_{1}}{\partial \bar{r}}\right|_{\bar{z}=\bar{h}}}{C a \bar{\mu}}
\end{gathered}
$$

For any scenario, the vertically averaged, horizontal velocity will be between the above limits.

Now that the horizontal velocity has been determined, the governing equation for the droplet height can be derived using a material balance, yielding a partial differential equation for the droplet height as a function of horizontal position and time.

$$
\frac{\partial \bar{h}}{\partial \bar{t}}=-\bar{E}(\bar{r})-\frac{1}{\bar{r}} \frac{\partial}{\partial \bar{r}}\left[\bar{r} \bar{h} \tilde{v_{r}}\right]
$$

Where $\bar{E}(\bar{r})=x_{1} \frac{E_{1}{ }^{\star}}{E_{2}{ }^{\star}}+\left(1-x_{1}\right)$. There are two contributions to the change in height. The first is a decrease due to evaporation of the volatile liquid. The second is a surface tension driven flow term.

The solid volume fraction, $\phi$ can be similarly derived to follow from

$$
\frac{\partial \phi}{\partial \bar{t}}=\frac{\phi \bar{E}(\bar{r})}{\bar{h}}-\tilde{v_{r}} \frac{\partial \phi}{\partial \bar{r}}+\frac{P e_{p}^{-1}}{\bar{r} \bar{h}} \frac{\partial}{\partial \bar{r}}\left[\bar{r} \bar{h} \frac{\partial \phi}{\partial \bar{r}}\right]
$$

Where the Péclet number, $P e_{p}$, is defined as $P e_{p}=\frac{R^{2} E_{2}{ }^{\star}}{H D_{p}}$.

There are three contributions to the change in solid volume fraction. An increase, due to evaporation of the volatile liquid, a decrease due to convection from regions of smaller volume fraction and a spatially variable diffusion.

A means to track the evolving liquid composition is also necessary. On a solid free basis, one obtains

$$
\frac{\partial x_{1}}{\partial \bar{t}}=\frac{x_{1}\left(\bar{E}^{\prime}(\bar{r})+\bar{D}_{p}(\bar{r})\right)}{\bar{h}(1-\phi)}-\tilde{\overline{v_{r}}} \frac{\partial x_{1}}{\partial \bar{r}}+\frac{P e_{l l}^{-1}}{\bar{r} \bar{h}(1-\phi)} \frac{\partial}{\partial \bar{r}}\left[\bar{r} \bar{h}(1-\phi) \frac{\partial x_{1}}{\partial \bar{r}}\right]
$$

$$
\text { Where } \bar{E}^{\prime}(\bar{r})=\left[\left(x_{1}-1\right) \frac{E_{1}^{\star}}{E_{2}^{\star}}+\left(1-x_{1}\right)\right] \text { and } \bar{D}_{p}(\bar{r})=\frac{P e_{p}^{-1}}{\bar{r}} \frac{\partial}{\partial \bar{r}}\left[\bar{r} \bar{h} \frac{\partial \phi}{\partial \bar{r}}\right]
$$

There are three contributions to the liquid compositional change. The first is a decrease in mole fraction of component 1 , due to evaporation of component 1 and the increase in solid volume fraction. The second is an increase due to convection from areas of larger concentration. The third is a spatially variable, liquid-liquid diffusion contribution.

\subsection{Boundary \& Initial Conditions}

The governing equation for height is a fourth order partial differential equation. Four boundary conditions are hence required. The edge of the droplet has zero height and the horizontal velocity at this point is zero, to ensure that the droplet remains pinned. At the central boundary, the system is symmetrical $\left(\left.\frac{\partial \bar{h}}{\partial \bar{r}}\right|_{\bar{r}=0},\left.\frac{\partial \phi}{\partial \bar{r}}\right|_{\bar{r}=0},\left.\frac{\partial x_{1}}{\partial \bar{r}}\right|_{\bar{r}=0}=0\right)$ and there is zero flux across the centreline.

As the volatile liquid evaporates, the volume fraction of solid material increases. This 


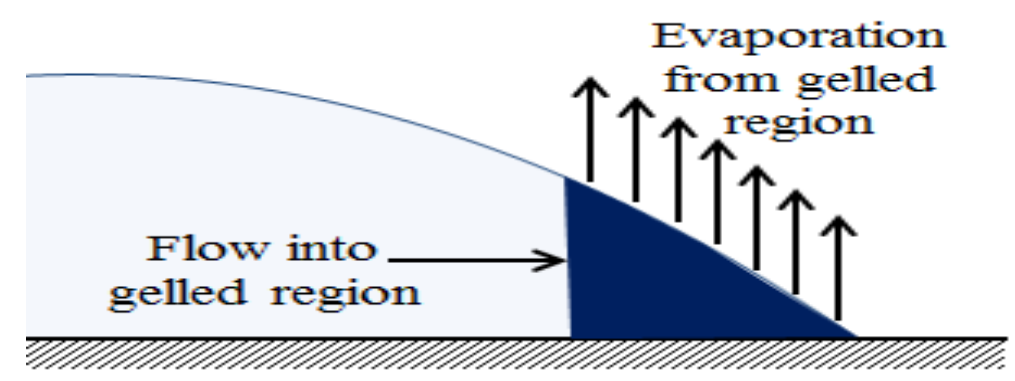

FIGURE 2. Sketch of the balance used to find the horizontal velocity at the position of the liquid/gel front. The flux into the gelled region is matched to the total evaporation rate, from the gelled region.

is most marked towards the edge of the droplet where the height is smallest. Soon, a close-packed or gelled region forms at the periphery, when $\phi=\phi_{\max }$. The inner most position of this region is termed the liquid/gel front, since its position changes with time. In the gelled region, the mole fraction of the liquid components may still change, provided evaporation occurs, but the height and solid volume fraction are fixed. The governing equations for height and solid volume fraction derived in section 2.3 are only applicable for the liquid domain, between the centre and the liquid/gel front. When this situation arises, the outer boundary conditions need to be changed. In this scenario the height is fixed at the liquid/gel front location, $\bar{r}_{f}$, and the horizontal velocity is found by equating the flux through the liquid/gel front with the liquid evaporated from within the gelled region as shown in figure 2. For close-packed solids this assumption is very good. We note that for polymer gels, shrinkage will occur and the inflow of liquid from the liquid domain might be insufficient to ensure the height is fixed in the gelled region. However, our present work focuses more on the final, rather than intermediary film profile. Therefore, for our purposes, whether a polymer gel would start to shrink immediately or after the entire droplet has gelled, would not be important.

This is a volumetric average and hence there is no need to include a rate of front progression term, $\frac{d \bar{r}_{f}}{d \bar{t}}$. The integral, in equation 2.33 , can be numerically evaluated at each time-step.

$$
\left.\overline{\tilde{v}}_{r}\right|_{\bar{r}=\bar{r}_{f}}=\frac{\left(1-f_{h}\right) \int_{\bar{r}_{f}}^{1} \bar{r} \bar{E}(\bar{r})}{\bar{r}_{f} \bar{h}_{f}}
$$

The partial differential equation for the liquid composition (equation 2.31) does not hold in the gelled region. It was previously assumed that volume fraction and height were functions of time and horizontal position. Instead the equation for the liquid composition in the gelled region becomes

$$
\frac{\partial x_{1}}{\partial \bar{t}}=\frac{x_{1} \bar{E}^{\prime}(\bar{r})}{\bar{h}\left(1-\phi_{\max }\right)}-\tilde{\overline{v_{r}}} \frac{\partial x_{1}}{\partial \bar{r}}+\frac{P e_{l l}^{-1}}{\bar{r} \bar{h}} \frac{\partial}{\partial \bar{r}}\left[\bar{r} \bar{h} \frac{\partial x_{1}}{\partial \bar{r}}\right]
$$

The notable difference, compared to equation 2.31, is that there is no contribution from the change in polymer volume fraction.

Initially, the droplet is assumed to have a spherical cap shape, $\bar{h}=1-\bar{r}^{2}$, and homogeneous distribution of solid material, at volume fraction $\phi_{0}$. The liquid is initially homogeneously distributed, with mole fraction ratio $x_{10}:\left(1-x_{10}\right)$. 


\subsection{Numerical Implementation}

A mixed scheme is chosen to solve the governing equation for height. Provided the timestep is sufficiently small, the height change will be slower than changes to the spatial derivatives of height. This approximation enables the partial differential equation, 2.29 to be linearised. This is then solved using Newton's method. For the solid volume fraction and the mole fraction of liquid component 1 , an implicit formulation is used.

\subsection{Groups to Examine}

The numerical solutions in this paper will consider the relative volatility of the two liquid components, their initial mixing ratio, viscosity ratio and surface tension ratio, as well as the magnitude of the solid and liquid-liquid Péclet numbers.

\section{Experimental Details}

The experiments use a flat, indium tin oxide (ITO) substrate. This consists of a $150 \mathrm{~nm}$ layer of conductive ITO, atop a $7 \mathrm{~mm}$ slide of glass. The substrates were bathed in acetone, followed by isopropanol, to pre-wash and remove any hydrocarbon residues and dust. A Litrex $80 \mathrm{~L}$ inkjet printer was used to jet droplets of various formulations onto each substrate. The inks consist of a volatile liquid, or mixture of volatile liquids, and a polyfluorene, light emitting polymer (LEP). The printer reports the jetting velocity, volume of the jetted droplet and the angle at which the ink leaves the inkjet head. The associated errors are also known.

Following jetting, the substrates were placed on a hot-plate, held at $80^{\circ} \mathrm{C}$. At this temperature, the polymer diffusion becomes negligible $\left(P e_{p} \sim 10^{2}\right)$ and the liquid-liquid diffusion in the liquid region $\left(P e_{l l} \sim 10^{-1}\right)$ is less dominant than at lower temperatures. In addition, the liquid-liquid diffusion will be hindered at the edge of the droplets, due to polymer gelation.

Once the evaporation of the volatile liquid(s) was complete, a Zygo white light interferometer was used to measure the final height profiles. The interferometer is capable of non-invasive measurements of height in the range $1 \mathrm{~nm}$ to $10000 \mu \mathrm{m}$.

We assume the maximal volume fraction of polymer is only a weak function of the liquid composition and can therefore be taken as fixed at 0.080, as for a typical lightemitting polymer with $\mathrm{MW}=10^{5}$, in methyl benzoate (Eales et al. $(2015 \mathrm{a})$ ). Table 2 shows the properties of the inks used in the experiments.

For droplets pinned at the edge, the final base width is $2 R$. Assuming a spherical cap initial shape, the initial droplet height, $H$ can be estimated from the initial volume of the droplet.

$$
H \sim \frac{2 V}{\pi R^{2}}
$$

Where $V$ is the initial droplet volume and $H$ and $R$ are the vertical and horizontal length scales of the jetted droplet. These enable the dimensional, experimental measurements of $h$ as a function of $r$ to be cast into dimensionless form, for direct comparison with other ink formulations. Note that for conciseness we do not report the $H$ and $R$ values for each experiment, however, in all cases the droplets are thin since $H^{2} / R^{2} \ll 1$. The final dried height of the film can be determined from the plots shown later in section 5 by computing $H \bar{h}$.

Evaporation from the region containing the gelled polymer, causes the polymer gel to shrink. In the experiments, we say that the final volume fraction of polymer, following complete evaporation of all liquid, is $\phi=\phi_{f}$. For the numerical results, the height at the 


$\begin{array}{cccccccc}\text { Ink } & \begin{array}{c}\text { Polymer conc. } \\ \left(\times 10^{-2} \mathrm{ml}^{-1}\right)\end{array} & \begin{array}{c}\text { Polymer } M W \\ \left(\times 10^{5} \mathrm{gmol} \mathrm{mol}^{-1}\right)\end{array} & \begin{array}{c}\text { Vol \% } \\ \text { liquid 1 }\end{array} & \begin{array}{c}\text { Vol \% } \\ \text { liquid 2 }\end{array} & x_{10} & \frac{E_{1}{ }^{\star}}{E_{2}{ }^{\star}} & \frac{\gamma_{1}{ }^{\star}}{\gamma_{2}{ }^{\star}} \\ \mathrm{A} & 1.0 & 1.0 & \mathrm{~N} / \mathrm{A} & 100 \mathrm{MB} & 0.00 & 1 & 1.00 \\ \mathrm{~B} & 1.0 & 1.0 & 25 \mathrm{AN} & 75 \mathrm{MB} & 0.28 & 10 & 0.93 \\ \mathrm{C} & 1.0 & 1.0 & 50 \mathrm{AN} & 50 \mathrm{MB} & 0.54 & 10 & 0.93 \\ \mathrm{D} & 1.0 & 1.0 & 75 \mathrm{AN} & 25 \mathrm{MB} & 0.78 & 10 & 0.93 \\ \mathrm{E} & 1.0 & 1.0 & \mathrm{~N} / \mathrm{A} & 100 \mathrm{AN} & 1.00 & 1 & 1.00 \\ \mathrm{~F} & 1.0 & 1.0 & 50 \mathrm{OX} & 50 \mathrm{MB} & 0.51 & 50 & 0.77 \\ \mathrm{G} & 1.0 & 2.0 & 50 \mathrm{AN} & 50 \mathrm{MB} & 0.54 & 10 & 0.93 \\ \mathrm{H} & 1.0 & 2.0 & 50 \mathrm{AN} & 50 \mathrm{~PB} & 0.61 & 8 & 1.19 \\ \mathrm{I} & 1.0 & 2.0 & 10 \mathrm{AN} & 90 \mathrm{~PB} & 0.15 & 8 & 1.19\end{array}$

TABLE 2. The table shows the constituents of each ink. For the liquids, the abbreviations are $\mathrm{MB}=$ methyl benzoate, $\mathrm{AN}=$ anisole, $\mathrm{OX}=\mathrm{o}$-xylene \& $\mathrm{PB}=$ pentyl benzene. The evaporation rates were measured by mass loss from a petri dish at room temperature. The surface tension ratios are calculated from measurements tabulated by Jasper (Jasper (1972)) at $80^{\circ} \mathrm{C}$

position of consideration, is fixed when the polymer volume fraction reaches the gelation point, $\phi=\phi_{\max }$. It does not take into account the shrinkage of the gel $\left(\phi_{f} \neq \phi_{\max }\right)$. To enable comparison, the experimental height is plotted as $\phi_{f} \cdot \bar{h} / \phi_{\max }$ against $\bar{r}$. To determine the final polymer volume fraction, $\phi_{f}$, in each experiment, we match the polymer quantity obtained from integration of the experimental profiles, to the initial polymer quantity.

$$
\phi_{f}=\frac{V \phi_{0}}{0.5 \int_{-R}^{R} 2 \pi r h d r}
$$

\section{Numerical Results \& Interpretation}

For conciseness, the results shown in sections 4.1-4.6 are for the first horizontal velocity limit, explained in section 2.3. This corresponds to the condition of zero-shear stress on the droplet surface. Comparative results are shown for the second horizontal velocity limit, a dominant Marangoni flow, in section 4.7.

\subsection{Relative Volatility}

To examine the influence of the relative volatilities of the liquid components, the viscosity and surface tension ratios are set to unity. In addition, the convection dominated regime is initially considered, so that both the polymer and liquid-liquid Péclet numbers tend to infinity. The final film shape is shown in figure 3 as a function of relative volatility. It should be noted that the case of $\frac{E_{1}{ }^{\star}}{E_{2}{ }^{\star}}=1$ is equivalent to a single liquid system.

When the relative volatility is increased, the coffee-ring effect is hindered. The peak ring height, at the edge, is reduced and more material is deposited centrally. Soon after evaporation commences, the composition within the droplet depends on the horizontal position. This can be understood by examining the denominator in equation 2.31. At the edge of the droplet, the height of the droplet is very small, therefore the change in composition, due to the different evaporation rates of the two liquid components, is largest at this point. The edge becomes rich in the less volatile liquid and depleted in the more volatile liquid. Hence, the evaporation rate is smaller at the edge and larger at the centre, when compared to a single liquid system. The effect therefore, is a reduction in the strength of the outward capillary flow required to maintain an equilibrium, spherical 


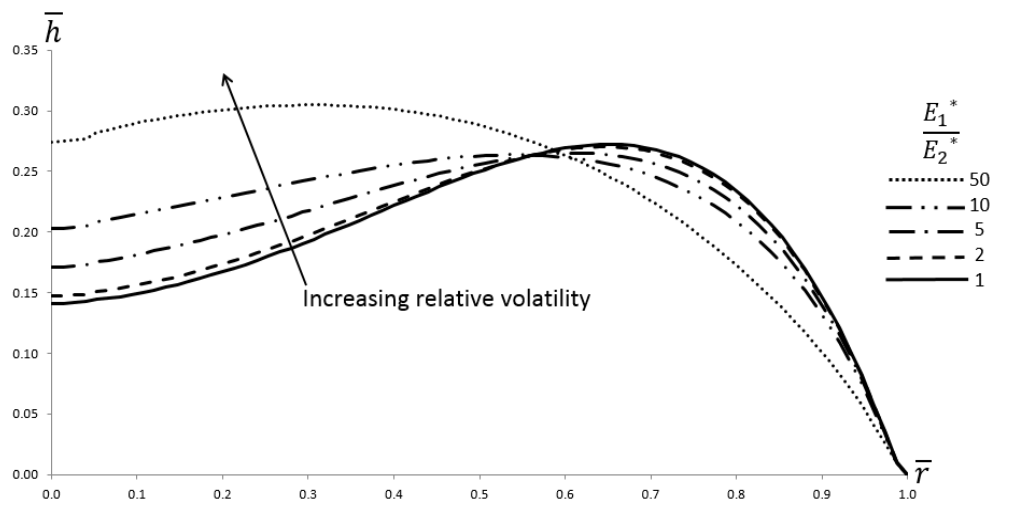

FiguRE 3. Numerical results for the final film shape as a function of the relative volatility. The results are shown for $C a=10^{-3}, \phi_{0}=0.032, \phi_{\max }=0.080, f_{h}=0.95, \mu_{1}{ }^{\star} / \mu_{2}{ }^{\star}=1$, $\gamma_{1}{ }^{\star} / \gamma_{2}{ }^{\star}=1, x_{10}=0.5, P e_{p} \rightarrow \infty$ and $P e_{l l} \rightarrow \infty$.

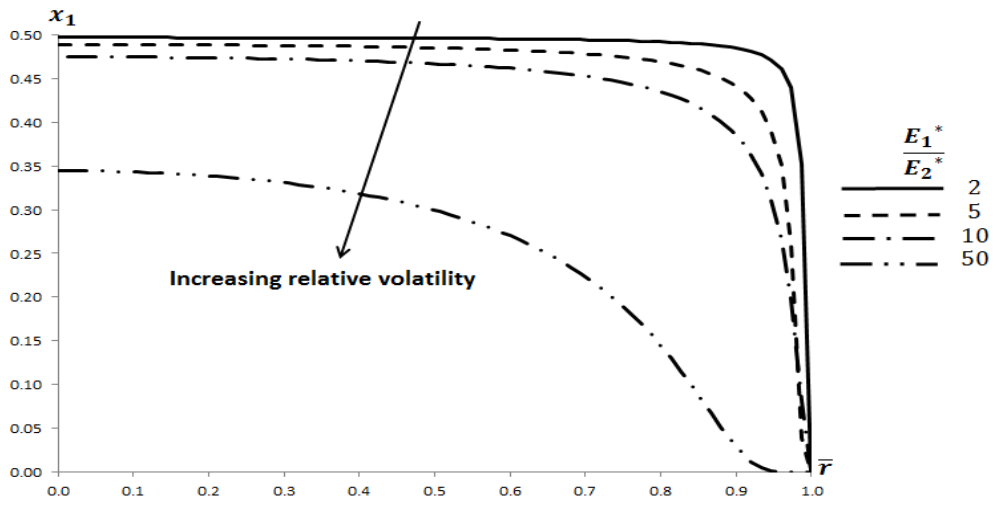

Figure 4. The liquid composition profile after a dimensionless time $\mathrm{t}=0.01$. The results are shown for $C a=10^{-3}, \phi_{0}=0.032, \phi_{\max }=0.080, f_{h}=0.95, \mu_{1}^{\star} / \mu_{2}{ }^{\star}=1, \gamma_{1}^{\star} / \gamma_{2}{ }^{\star}=1$, $x_{10}=0.5, P e_{p} \rightarrow \infty$ and $P e_{l l} \rightarrow \infty$.

cap shape. Thus, less polymer is transported towards the contact-line and less coffeering formation is observed. With increasing relative volatility, the less volatile rich region becomes less confined to the periphery, as can be seen in figure 4 . This reduces the coffee-ring effect further.

As a measure for the extent of the coffee-ring effect, we consider the ratio of the peak height of the deposited ring to the final height at the centre of the dried film. Figure 5 shows this measure as a function of the relative volatility. It shows that the coffee-ring effect is reduced when the relative volatility is increased. There are however diminishing returns at higher values of $\frac{E_{1}^{\star}}{E_{2}{ }^{\star}}$.

Depending on the desired final film shape, the relative volatility of the two liquids can be optimised. For example, if one wished to achieve as flat a film as possible, there will be an optimal relative volatility. Figure 3 suggests this would be at a relative volatility of greater than 10. If it was desired to obtain a spherical cap shape deposit, a much larger relative volatility would be appropriate.

\subsection{Liquid - Liquid Péclet Number}

In figure 6 , the film shape resulting from droplets with a variety of liquid-liquid Péclet numbers can be seen. At infinite Péclet number, diffusion is negligible. The mechanism 


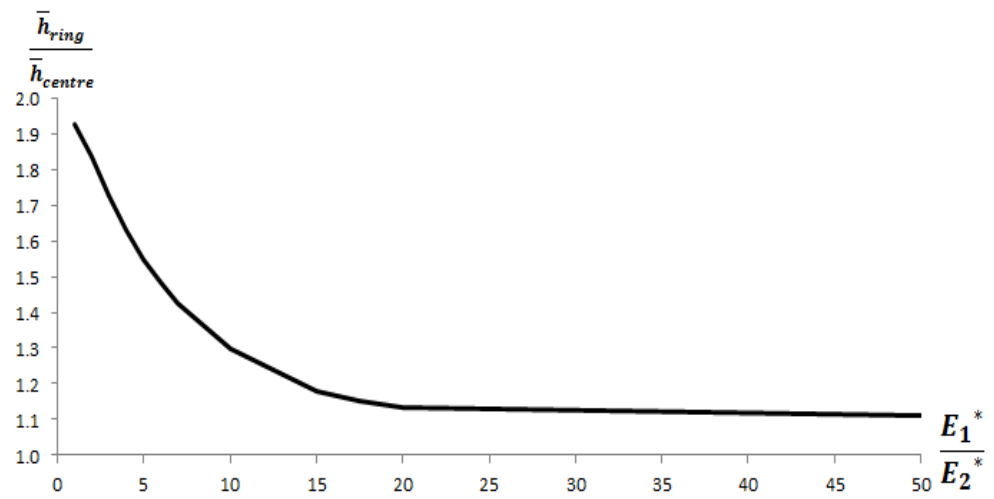

FiguRE 5. The ratio of the peak height of the deposited ring to the final central height, as a function of the relative volatility. The results are shown for $C a=10^{-3}, \phi_{0}=0.032, \phi_{\max }=0.080$, $f_{h}=0.95, \mu_{1}{ }^{\star} / \mu_{2}{ }^{\star}=1, \gamma_{1}{ }^{\star} / \gamma_{2}{ }^{\star}=1, x_{10}=0.5, P e_{p} \rightarrow \infty$ and $P e_{l l} \rightarrow \infty$.

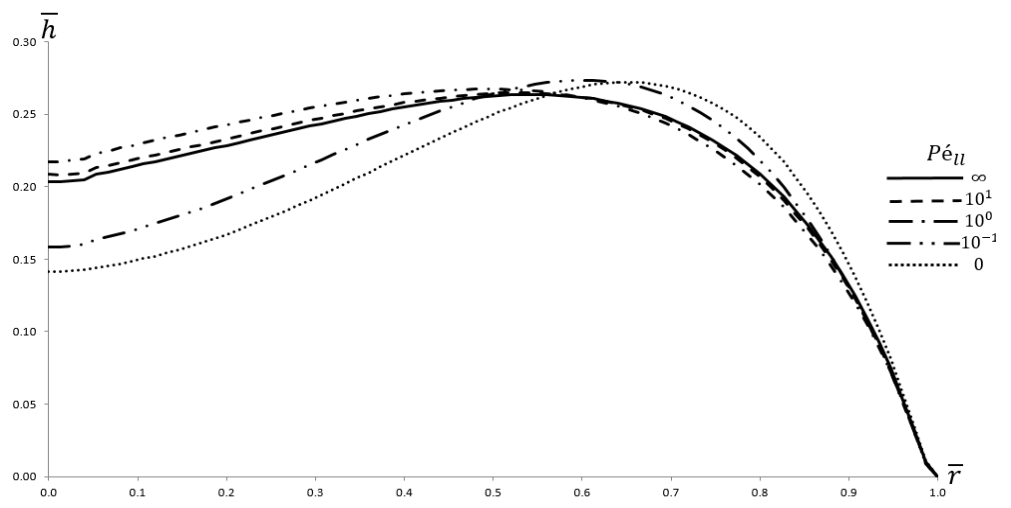

Figure 6. Final film shape as a function of the liquid-liquid Peclet number. The results are shown for $C a=10^{-3}, \phi_{0}=0.032, \phi_{\max }=0.080, f_{h}=0.95, E_{1}{ }^{\star} / E_{2}{ }^{\star}=10, \mu_{1}{ }^{\star} / \mu_{2}{ }^{\star}=1$, $\gamma_{1}^{\star} / \gamma_{2}{ }^{\star}=1, x_{10}=0.5$ and $P e_{p} \rightarrow \infty$.

outlined in section 4.1 occurs and the coffee-ring effect is reduced when compared to a single liquid system or a system in which the liquid volatilities are matched. In the presence of weak liquid-liquid diffusion, i.e. $P e_{l l}=10^{1}$, liquid material is redistributed within the droplet. The result is that the less volatile rich region at the edge diminishes, due to the increased presence of the more volatile liquid. Subsequently, there will be a larger evaporation rate near the edge, compared to the $P e_{l l} \rightarrow \infty$ case. It will take less time for the polymer to reach the gelation volume fraction and so there is less time for the outward Capillary flow to convect polymer, before the height is fixed by polymer gelation. The coffee-ring effect is further reduced, albeit only slightly.

The optimal film shape improvement is achieved at approximately $P e_{l l}=1$. Below this, diffusion dominates and causes equilibration in liquid composition throughout the droplet. This removes the mechanism for the improvement in film shape by ensuring a spatially uniform evaporation profile across the droplet surface. At $P e_{l l}^{\prime}=0$, the system essentially behaves like a single liquid system but with an evaporation rate, that is initially the midpoint of $E_{1}{ }^{\star}$ and $E_{2}{ }^{\star}$, and gradually decreases with time, as the mole fraction of more volatile liquid decreases. 


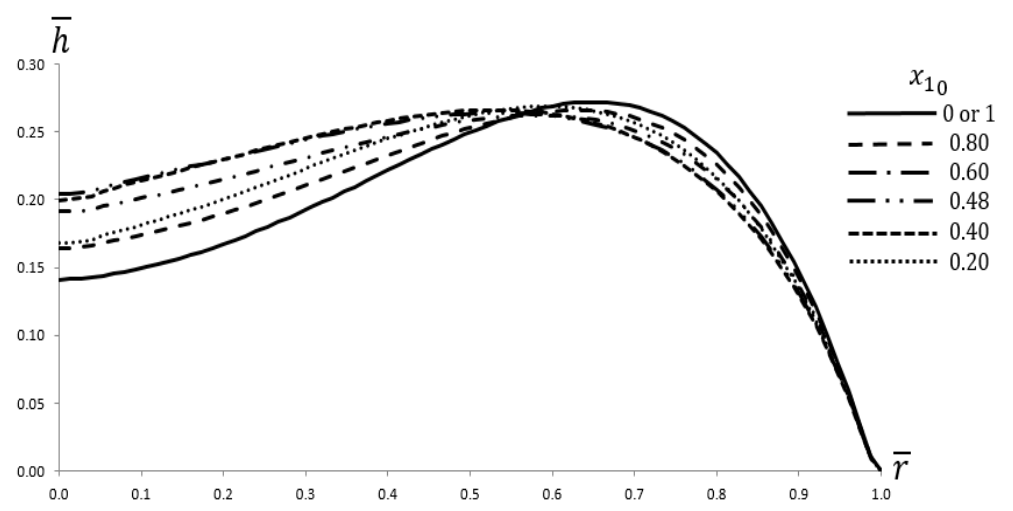

Figure 7. Final film shape as a function of the initial mole fraction of liquid 1 . The results are shown for $C a=10^{-3}, \phi_{0}=0.032, \phi_{\max }=0.080, f_{h}=0.95, E_{1}{ }^{\star} / E_{2}{ }^{\star}=10, \mu_{1}{ }^{\star} / \mu_{2}{ }^{\star}=1$, $\gamma_{1}^{\star} / \gamma_{2}{ }^{\star}=1, P e_{p} \rightarrow \infty$ and $P e_{l l} \rightarrow \infty$

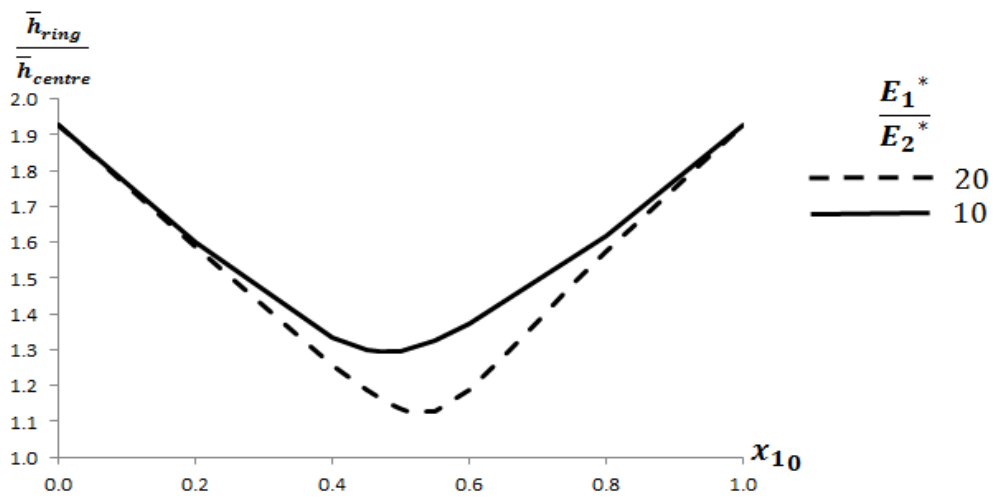

FIGURE 8. The ratio of the peak height of the deposited ring to the final central height, as a function of the initial mole fraction of liquid 1 and the relative volatility. The results are shown for $C a=10^{-3}, \phi_{0}=0.032, \phi_{\max }=0.080, f_{h}=0.95, \mu_{1}{ }^{\star} / \mu_{2}{ }^{\star}=1, \gamma_{1}{ }^{\star} / \gamma_{2}{ }^{\star}=1, P e_{p} \rightarrow \infty$ and $P e_{l l} \rightarrow \infty$.

\subsection{Initial Liquid Composition}

The influence of initial liquid composition on final film shape, for a system in which the viscosities and surface tensions of the two liquid components are matched, is considered. The trend with surface tension ratio is discussed later in section 4.5. It should be noted that the initial liquid composition has an influence on this trend also. In figure 7, the final shape is shown for a variety of initial mixtures, with a relative volatility of 10 and for a polymer content $\phi_{0}=0.032$ and $\phi_{\max }=0.080$.

At a fixed relative volatility and polymer content, there is an optimal initial mixing ratio which gives rise to the most improvement in film shape. In figure 8 , the peak ring to final central height ratio is plotted as a function of the initial mole fraction of the more volatile liquid. When the initial mole fraction is 0 or 1 the system is essentially a single liquid problem and no improvement in film shape, due to the relative volatility mechanism occurs. In this example, the optimal initial composition is $x_{1 \text { opt }}=0.48$ for a relative volatility of 10 and $x_{1 \text { opt }}=0.54$ for a relative volatility of 20 .

The initial and maximum volume fractions of the polymer are also important. In figure 9, the final shape is shown as a function of the initial liquid composition, at a much smaller polymer volume fraction ratio, $\phi_{0} / \phi_{\max }$, with $\phi_{0}=0.0032$ and $\phi_{\max }=0.1600$. 


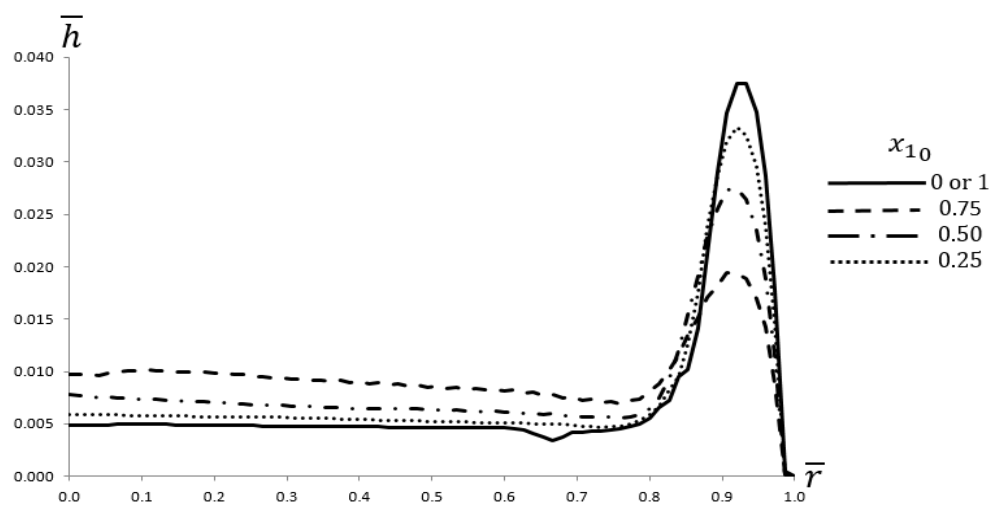

Figure 9. Final film shape as a function of the initial mole fraction of liquid 1. The results are shown for $C a=10^{-3}, \phi_{0}=0.0032, \phi_{\max }=0.160, f_{h}=0.95, E_{1}^{\star} / E_{2}^{\star}=10, \mu_{1}^{\star} / \mu_{2}^{\star}=1$, $\gamma_{1}^{\star} / \gamma_{2}{ }^{\star}=1, P e_{p} \rightarrow \infty$ and $P e_{l l} \rightarrow \infty$

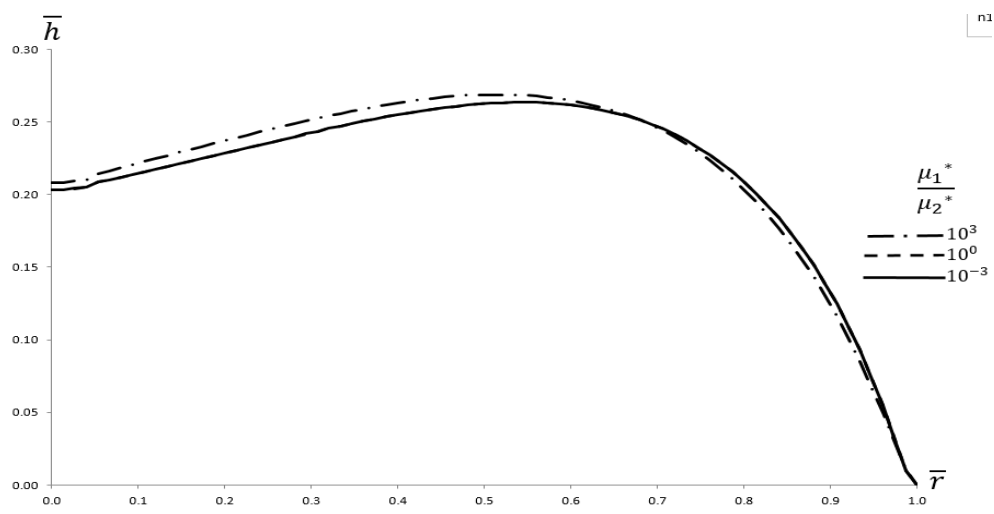

Figure 10. Final film shape as a function of the viscosity ratio. The results are shown for $C a=10^{-3}, \phi_{0}=0.032, \phi_{\max }=0.080, f_{h}=0.95, E_{1}{ }^{\star} / E_{2}{ }^{\star}=10, \gamma_{1}{ }^{\star} / \gamma_{2}{ }^{\star}=1, x_{10}=0.5$, $P e_{p} \rightarrow \infty$ and $P e_{l l} \rightarrow \infty$.

The evaporation suppression factor for these simulations, $f_{h}$, is set to unity, in order to avoid a polymer volume fraction complication to be discussed later, in section 4.7. As the polymer volume fraction ratio is decreased, the optimal initial mole fraction of the more volatile component increases. For smaller polymer contents, a larger $x_{10}$ causes polymer gelation to occur much sooner. There is hence less time for the outward capillary flow to drive coffee-ring formation before the height becomes fixed.

\subsection{Viscosity Ratio}

As discussed in section 2.2, the Capillary number is small: surface tension dominates over viscous effects. For this reason, the viscosity ratio is unimportant. It does alter the horizontal velocity slightly, but it does not alter the magnitude of the horizontal velocity and so the resultant effect on film shape is marginal. Figure 10, shows the film shape for various values of the viscosity ratio, which only results in an appreciable change to the final film, when it is very large, i.e. the viscosity of the more volatile component is three orders of magnitude larger than the viscosity of the less volatile liquid. This is because the more volatile rich central region has a reduced mobility, so the outward Capillary flow is hindered. Situations with such a large differential in liquid viscosity are probably physically unreasonable. 


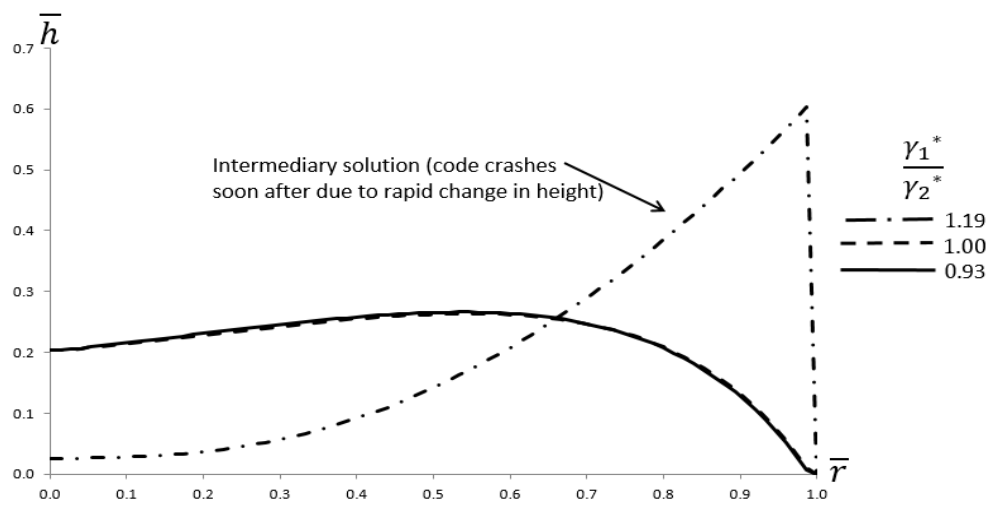

FiguRE 11. Final film shape as a function of the surface tension ratio. The results are shown for $C a=10^{-3}, \phi_{0}=0.032, \phi_{\max }=0.080, f_{h}=0.95, E_{1}{ }^{\star} / E_{2}{ }^{\star}=10, \mu_{1}{ }^{\star} / \mu_{2}{ }^{\star}=1, x_{10}=0.5$, $\mathrm{Pe}_{p} \rightarrow \infty$ and $\mathrm{Pe} l l \rightarrow \infty$.

\subsection{Surface Tension Ratio}

By using typical values for the surface occupancy of molecules in equation 2.6, quoted by Suri and Ramakrishna (Suri \& Ramakrishna (1968)), we observe no appreciable difference in predicted film shape between using a linear fit and the non-linear model of equation 2.6 , for the surface tension of the binary liquid mixture.

There are two different situations. Either the more volatile liquid has a higher surface tension than the less volatile liquid, $\frac{\gamma_{1}}{\gamma_{2}}>1$ or it has a lower surface tension, $\frac{\gamma_{1}}{\gamma_{2}}<1$. As can be seen in figure 11, there is little difference in film shape in the latter case. When the more volatile liquid has a higher surface tension, however, we observe some interesting phenomena. There is a rapid decrease in height towards the centre of the droplet and a large increase in height, towards the periphery. This leads to a crash in the code, which cannot track the height of the droplet accurately. It is believed the reason for this, is an instability in the height caused by the compositional and resulting surface tension gradients within the droplet driving a large internal flow. This will be investigated further in section 6 .

\subsection{Solid Péclet Number}

The results shown up to this point consider the case of infinite polymer Péclet number. We extend this to consider weak diffusion. When the Péclet number decreases, diffusion of the polymer becomes more appreciable, and there is inward diffusion of polymer, down the gradient in polymer volume fraction. This results in a decrease to the coffeering effect. Less material is deposited towards the edge and more is deposited centrally. Figure 12 shows the final film shape as a function of the polymer Péclet number.

\subsection{Comparison of the two horizontal velocity limits}

The results in sections 4.1-4.6 consider only the first horizontal velocity limit, stated in equation 2.26. It is important to consider what happens if the horizontal velocity is closer to the second limit, given in equation 2.28. In any situation, the actual horizontal velocity will be between these two extremes.

The final film shape is shown in Figure 13 for a typical scenario with surface tension ratio $\gamma_{1}^{\star} / \gamma_{2}^{\star}<1$. 


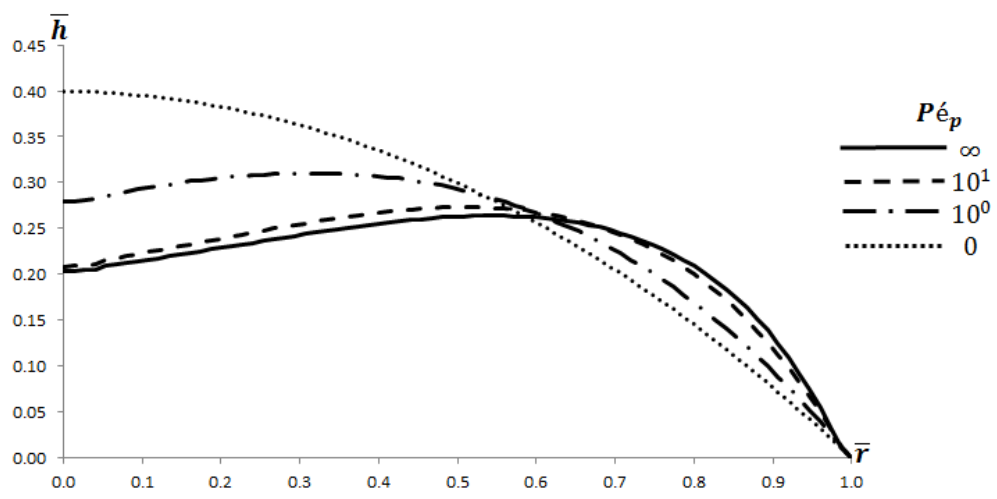

Figure 12. Final film shape as a function of the polymer Peclet number. The results are shown for $C a=10^{-3}, \phi_{0}=0.032, \phi_{\max }=0.080, f_{h}=0.95, E_{1}{ }^{\star} / E_{2}{ }^{\star}=10, \mu_{1}^{\star} / \mu_{2}{ }^{\star}=1, \gamma_{1}^{\star} / \gamma_{2}{ }^{\star}=1$, $x_{10}=0.5$ and $\mathrm{Pe}_{l l} \rightarrow \infty$.

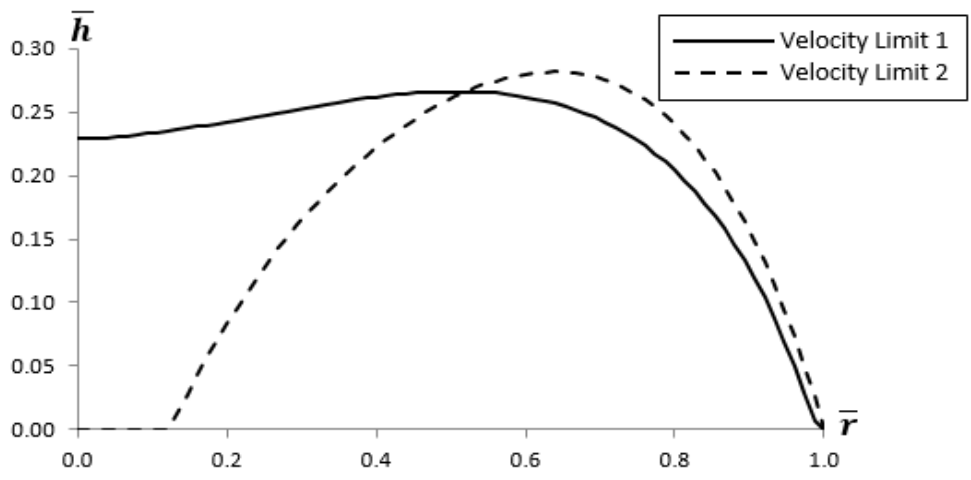

Figure 13. Final film shape for the two limits of horizontal velocity. The results are shown for $C a=10^{-3}, \phi_{0}=0.032, \phi_{\max }=0.080, f_{h}=1.0, E_{1}{ }^{\star} / E_{2}{ }^{\star}=10, \mu_{1}{ }^{\star} / \mu_{2}{ }^{\star}=1, \gamma_{1}{ }^{\star} / \gamma_{2}{ }^{\star}=0.9$, $x_{10}=0.5, P e_{p} \rightarrow \infty$ and $P e_{l l} \rightarrow \infty$.

In the second horizontal velocity limit, a strong outward Marangoni flow convects solid to the edge and results in an enhanced coffee-ring formation.

It is important to note that a numerical solution is also not possible for the second horizontal velocity limit, when the surface tension ratio $\gamma_{1}{ }^{\star} / \gamma_{2}{ }^{\star}>1$. It is thought that this is due to the onset of an instability and will be examined further in the linear stability analysis of section 6 . We note that prior to the crash in the code, the solids fraction falls dramatically near the edge. This could be consistent with a strong inward Marangoni flow and associated depinning of the contact line, resulting in a deposit only at the droplet centre, as widely reported in literature, one example being Babatunde et al. (2013).

\section{Experimental Results \& Discussion}

\subsection{Initial Liquid Composition}

Figure 14 shows the film shape resulting from inks A-E. The initial composition appears to have a subtle effect. This is because liquid-liquid diffusion is significant and the tendance is to equilibrate the liquid composition within the droplet, thus removing or at least reducing the extent of the relative volatility mechanism proposed in section 4.1. Previous works, for example Fischer (Fischer (2002)) and Eales et al. (Eales et al. 


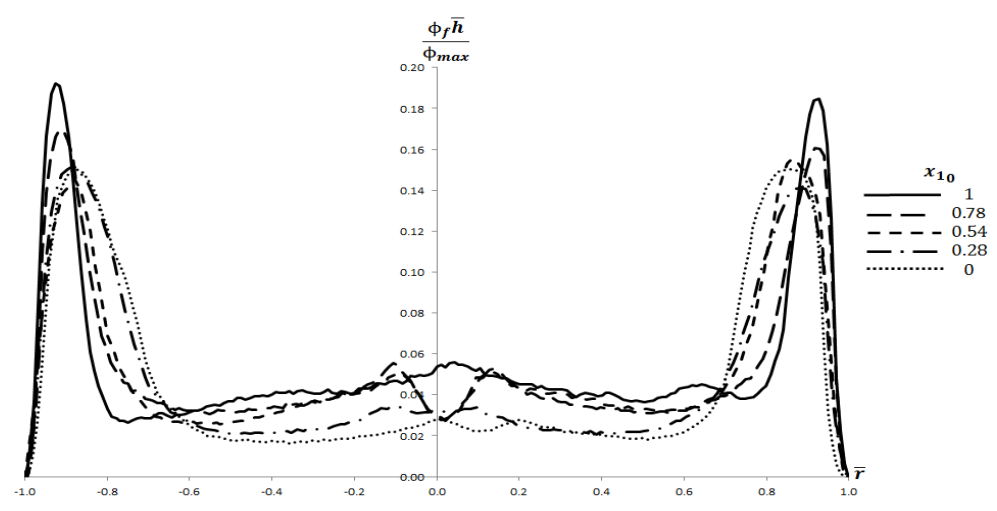

FiguRe 14. Experimental results for the final film profile from anisole / methyl benzoate inks $\mathrm{A}-\mathrm{E}$, as a function of the initial mole fraction of anisole. The properties of inks A-E can be found in table 2 .

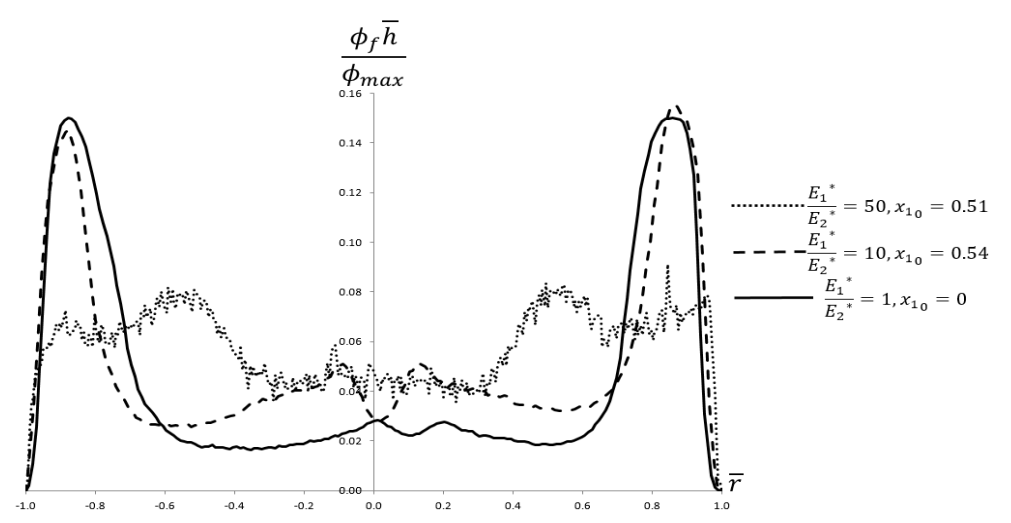

FIGURE 15. Experimental results for the final film profile as a function of the relative volatility.

The inks shown are A, C and F. The properties of these inks can be found in table 2 .

$(2015 a)$ ), have shown that coffee-ring formation is accentuated by edge enhanced evaporation. Despite the Capillary number being small in both cases, the evaporation rate profiles across droplets of methyl benzoate and anisole, could be different. This could explain the repeatable difference in the final shapes for methyl benzoate and anisole, shown in Figure 14. It should be noted that the error bars are not shown in Figure 14, for clarity, but the experimental error is $\pm 10 \%$ by height and cannot explain the trend.

\subsection{Relative Volatility}

The plots in Figure 15, represent the final film profiles for inks A, C \& F. There appears to be an improvement in film shape as the relative volatility is increased. Less material is deposited at the edge and more is deposited at the centre. The extent of coffee-ring formation is reduced. As the relative volatility is increased, we expect there to be a larger differential in evaporation rate between the centre and edge.

This agrees with the work of Teichler et al. (Teichler et al. (2013)), who found that the surface roughness of films decreases when the difference in liquid boiling points is increased.

It was previously argued that the capillary number is very small, such that surface tension effects dominate. This was true for the majority of droplets under consideration. In the case of ink F, however, the droplet wetted the ITO much better and the capillary 


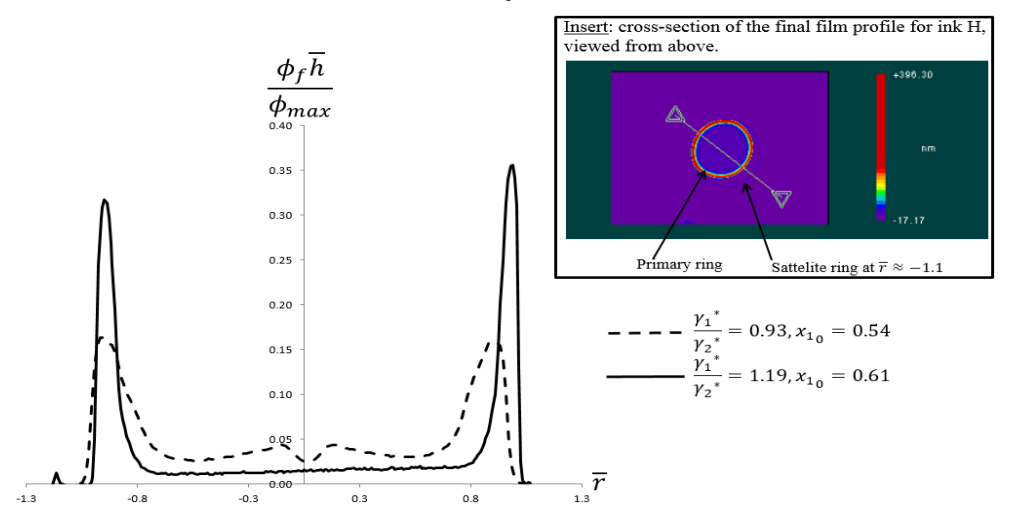

FiguRE 16. Experimental results for the final film profile as a function of the surface tension ratio. The insert shows the white-light interferometer data for the anisole / pentyl benzene droplet (ink $\mathrm{H}$ ). A primary coffee-ring was observed, with a satellite at a seemingly random orientation. The inks shown are G and $\mathrm{H}$. The properties of these inks can be found in table 2 .

number was order $10^{-1}$. Whilst surface tension effects are still important, this could have a small contribution to the improvement in shape observed in Figure 15, as predicted by our previous work (Eales et al. $(2015 a))$.

\subsection{Surface Tension Ratio}

In Figure 16, the final film shape is shown for the anisole / methyl benzoate mixture of ink $\mathrm{G}$ and for the anisole / pentyl benzene mixture of ink H. For the former, the surface tension ratio $\frac{\gamma_{1}^{\star}}{\gamma_{2}{ }^{\star}}=0.93$ at $80^{\circ} \mathrm{C}$ and the resultant film shape is similar to those previously observed. For the latter, however, the surface tension ratio, $\frac{\gamma_{1}{ }^{\star}}{\gamma_{2}{ }^{*}}$, is 1.19 and an extreme coffee-ring formation results. There is minimal material deposited centrally, within the droplet and a region of intense deposition at the periphery. These results were repeatable. This would appear to be consistent with the dynamics of the numerical solution under these conditions (see Figure 11). Interestingly, the cross-section for $\frac{\gamma_{1}^{\star}}{\gamma_{2}{ }^{\star}}=1.19$ in Figure 16 shows a larger coffee-ring height at one side and a smaller one at the other, with what appears to be a satellite coffee-ring (see the cross-section of the final film profile, inserted to the top right hand side of Figure 16). Looking at the 3D deposit, this occurred repeatably but at a seemingly random orientation. The secondary crescent shaped ring of much smaller height, suggests that there is an overspill of the droplet, beyond the original confines of the edge. The instability and the associated strong outward flow is a possible cause of this phenomenon. Henceforth we will say that an instability has occurred if we observe a satellite coffee-ring.

\section{A linear stability analysis of the lubrication model}

\subsection{Description and methodology}

We wish to determine any situations in which the ink droplets, printed onto our substrates, will have an instability in the height, due to the growth of perturbations. In the eventuality that they become unstable, it is desired to understand the mechanism driving the growth of perturbations. The presence of polymer is ignored, as are the polymer and liquid-liquid diffusion, because these are likely to have a stabilising effect. A detailed analysis of how to avoid instabilities may wish to include these also, but this falls beyond the scope of the present work. The linear stability analysis is performed on equations 
2.29 and 2.31, using base states $\bar{h}_{b}$ and $\bar{x}_{1, b}$ and small perturbation terms $\hat{\bar{h}}$ and $\hat{\bar{x}}_{1}$, such that

$$
\begin{gathered}
\bar{h}=\bar{h}_{b}+\hat{\bar{h}} e^{(i k \bar{r}+\omega \bar{t})} \\
\bar{x}_{1}=\bar{x}_{1, b}+\hat{\bar{x}}_{1} e^{(i k \bar{r}+\omega \bar{t})}
\end{gathered}
$$

In equations 6.1 and $6.2, k$ is the wavenumber and $\omega$ is the growth rate. To determine the stability, it is necessary to determine the sign of $\omega$. Expressions can be found for each of the terms in the partial differential equations 2.29 and 2.31, ignoring all nonlinear perturbation terms. The expressions resulting from consideration of the real part of perturbation terms alone, can be summarised as

$$
\omega\left(\begin{array}{c}
\hat{\bar{h}} \\
\hat{\bar{x}}_{1}
\end{array}\right)=\left(\begin{array}{cc}
A_{11} & A_{12} \\
A_{21} & A_{22}
\end{array}\right)\left(\begin{array}{c}
\hat{\bar{h}} \\
\hat{\bar{x}}_{1}
\end{array}\right)
$$

Where the matrix coefficients $A_{i j}$ represent the coefficient of growth for perturbation $i$, with respect to the introduced perturbation $j$. For conciseness, the full form of these matrix coefficients are reported in supporting information. Generally, the coefficients $A_{i j}$ are a function of $C a, k, \bar{r},\left(\frac{E_{1}^{\star}}{E_{2}{ }^{\star}}-1\right),\left(\frac{\gamma_{1}^{\star}}{\gamma_{2}{ }^{\star}}-1\right), \bar{x}_{1,0}, \bar{h}(\bar{r})$ and $\bar{x}_{1}(\bar{r})$.

Equation 6.3 is solved for $\omega$. Two solutions are obtained and the system will be unstable, if either solution is positive, at any physically realisable wavenumber, $k$.

It is known that the height at the edge of the droplet is zero. Hence, only discrete values of $k=\frac{m \pi}{2}$ are physically meaningful for the system, where $m$ is a positive integer.

\subsection{Solution methodology}

In practice, the capillary number is small. The base case is therefore taken to be $C a=$ $10^{-3}$, throughout this analysis. We consider the situation, soon after evaporation has commenced. The droplet has a spherical cap shape $\bar{h}=1-\bar{r}^{2}$. For any $x_{10}$, the liquid composition profile $\bar{x}_{1}(\bar{r})$ is taken from the numerical solution after one time-step (with $\left.\Delta \bar{t}=10^{-3}\right)$.

As the wavenumber $k$ gets larger, the solutions for $\omega$ tend to a steady state value. For this reason, we only solve for the first 50 discrete values of $k$.

For any given parameter values the resulting $\omega$ as a function of $k$ is plotted at various radial positions, $r$. Any positive value for $\omega$ indicates an instability and hence a global stability locus can be generated as a function of $\frac{\gamma_{1}^{\star}{ }^{\star}}{\gamma_{2}}, \frac{E_{1^{\star}}}{E_{2}{ }^{\star}}$ and $x_{10}$.

\subsection{Results $\&$ Interpretation}

For conciseness, the results shown in sections 6.3.1 - 6.3.5 and discussed below, consider the first horizontal velocity limit, outlined in section 2.3 and equation 2.26. This corresponds to the condition of zero-shear stress on the free surface. Comparative results are shown in section 6.3.6, for the second limit (equation 2.28), a dominant Marangoni flow.

One solution for $\omega$ is always negative. Hence, only the one solution for $\omega$, termed $\omega_{2}$, is shown in subsequent figures. The stability results for the binary liquid droplets depend on the position under consideration, $\bar{r}$, the surface tension ratio, $\frac{\gamma_{1}^{\star}{ }^{\star}{ }^{\star}}{\gamma^{*}}$, the initial mole fraction of the more volatile liquid, $x_{10}$ and the relative volatility, $\frac{E_{1}{ }^{\star}}{E_{2}{ }^{\star}}$. When considering the global stability, the situation under consideration is only stable if it is stable at every location within the droplet. For this reason, the linear stability calculations must be repeated many times. 


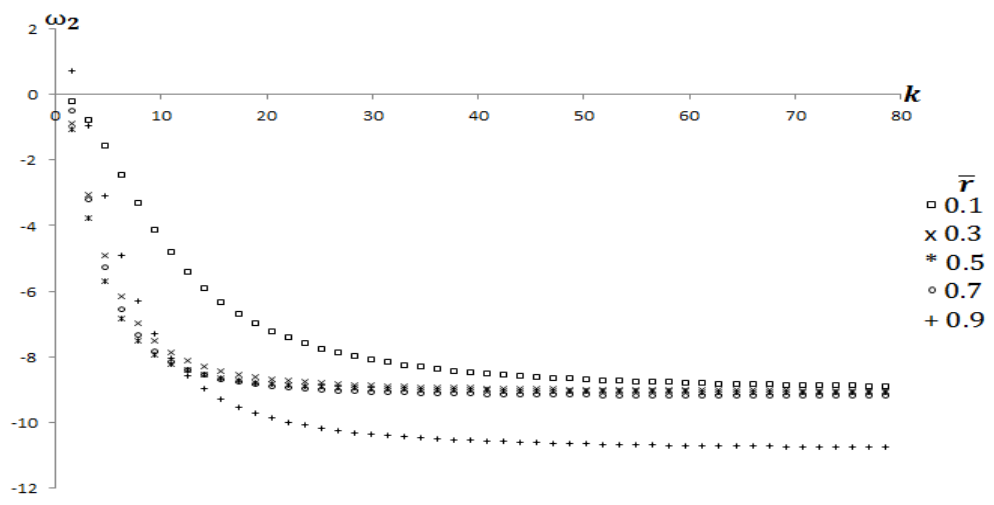

FiguRE 17. Stability results as a function of the position within the droplet. The results are shown for $C a=10^{-3}, E_{1}{ }^{\star} / E_{2}{ }^{\star}=10, \gamma_{1}{ }^{\star} / \gamma_{2}{ }^{\star}=0.5$ and $x_{10}=0.5$.

\subsubsection{Trend with position within the droplet}

The stability results are highly sensitive to the chosen position within the droplet. For a fixed initial mole fraction of the more volatile component, relative volatility and surface tension ratio, there is a critical, $\bar{r}$, that is most likely to give rise to an instability. This critical position is a function of the surface tension ratio. Figure 17 shows the stability results as a function of position, for the case of $\frac{\gamma_{1}^{\star}}{\gamma_{2^{\star}}}=0.5$.

When the surface tension ratio, $\frac{\gamma_{1}{ }^{\star}}{\gamma_{2}{ }^{\star}}<1$, the critical position is typically towards the edge of the droplet, at approximately $\bar{r}=0.99$. When the surface tension ratio, $\frac{\gamma_{1}{ }^{\star}}{\gamma_{2}{ }^{\star}}>1$, the critical position is further towards the centre of the droplet, at approximately $\bar{r}=0.1$.

\subsubsection{Trend with surface tension ratio}

The stability results are generally a strong function of the surface tension ratio. Figure 18 shows the trend with surface tension ratio for the scenario $\bar{r}=0.5, x_{10}=0.5, \frac{E_{1}{ }^{\star}}{E_{2^{*}}}=10$. Under these conditions, if the surface tension of the more volatile component is larger than the surface tension of the less volatile component (i.e. $\frac{\gamma_{1}{ }^{\star}}{\gamma_{2}{ }^{\star}}>1$ ), an instability will arise. Conversely, if the surface tension of the more volatile component is either directly matched to or less than the surface tension of the less volatile component (i.e. $\frac{\gamma_{1}^{\star}}{\gamma_{2}{ }^{\star}} \leqslant 1$ ), the situation is stable. This behaviour is however, by no means universal, since it depends on the values of $\bar{r}, x_{10}$ and $\frac{E_{1}^{\star}}{E_{2}{ }^{\star}}$.

\subsubsection{Trend with initial liquid composition}

The plot in Figure 19 shows the stability results for different $x_{10}$ when the surface tension ratio is less than 1 and in Figure 20 when the surface tension ratio is greater than 1 .

It should be noted that the previously stable case of $\frac{\gamma_{1}^{\star}}{\gamma_{2}{ }^{\star}}<1$ can become unstable if $x_{10}$ is sufficiently large. Furthermore, the previously unstable case of $\frac{\gamma_{1}^{\star}}{\gamma_{2}^{\star}}>1$ can become stable if $x_{10}$ is small enough.

In the general case, if $x_{10}$ is larger, the scenario under consideration is more likely to be unstable.

\subsubsection{Trend with relative volatility}

The trend with relative volatility is shown in Figure 21 for a surface tension ratio of $\frac{\gamma_{1}^{\star}}{\gamma_{2}{ }^{\star}}=0.5$. 


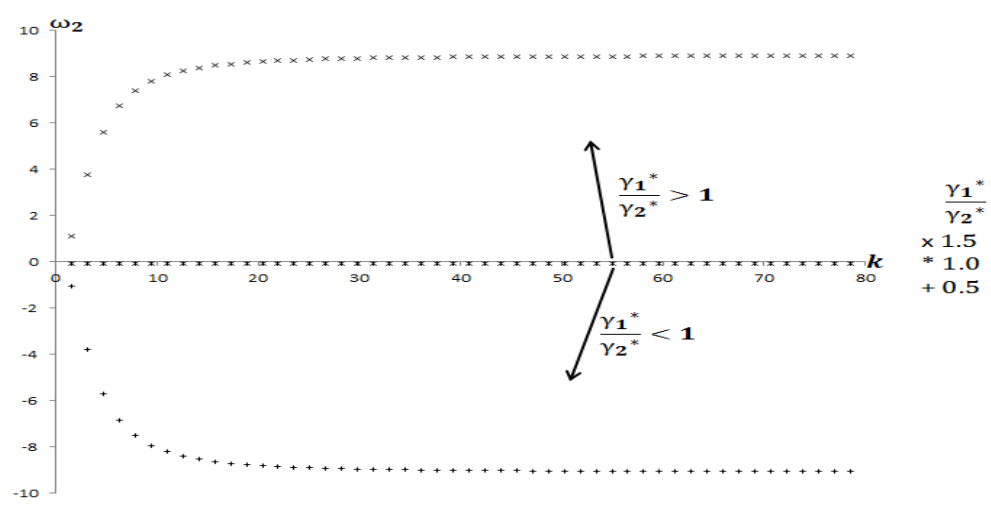

FiguRE 18. Stability results as a function of the surface tension ratio. The results are shown for $C a=10^{-3}, \bar{r}=0.5, E_{1}^{\star} / E_{2}^{\star}=10$ and $x_{10}=0.5$.

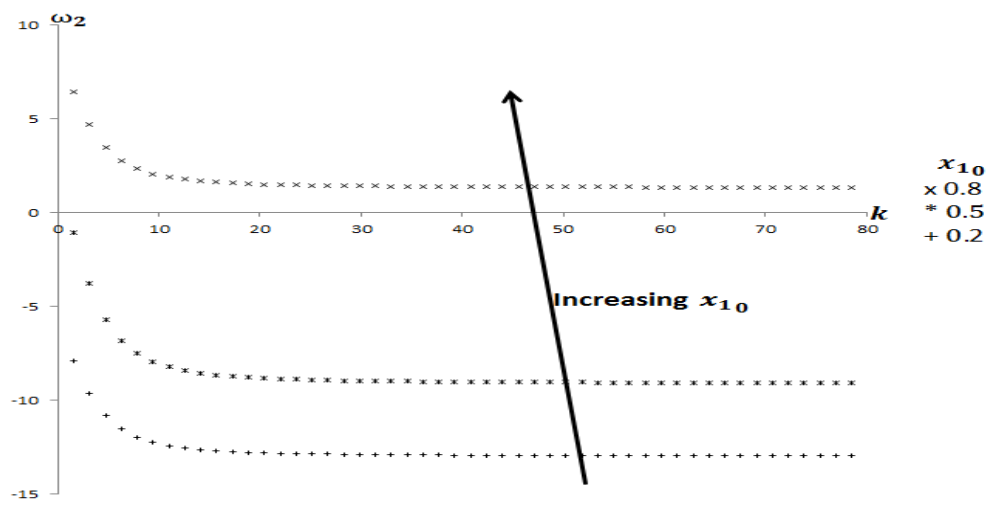

Figure 19. Stability results as a function of the initial mole fraction liquid 1 . The results are shown for $C a=10^{-3}, \bar{r}=0.5, E_{1}{ }^{\star} / E_{2}{ }^{\star}=10$ and ${\gamma_{1}}^{\star} / \gamma_{2}{ }^{\star}=0.5$.

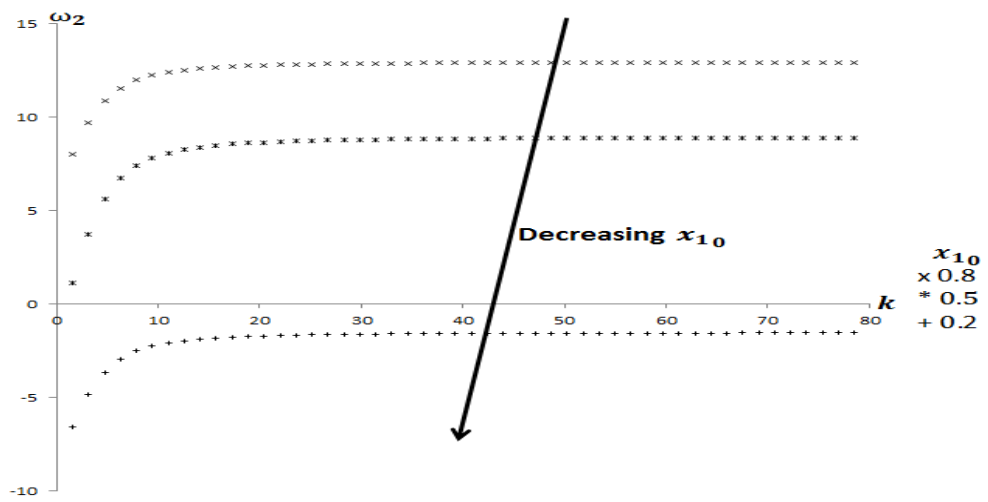

FIgURE 20. Stability results as a function of the initial mole fraction of liquid 1 . The results are shown for $C a=10^{-3}, \bar{r}=0.5, E_{1}{ }^{\star} / E_{2}{ }^{\star}=10$ and $\gamma_{1}{ }^{\star} / \gamma_{2}{ }^{\star}=1.5$.

In the general case, if the relative volatility is increased, binary liquid droplets are increasingly likely to be stable. This effect is more pronounced for $\frac{\gamma_{1}^{\star}}{\gamma_{2}{ }^{\star}}<1$. 


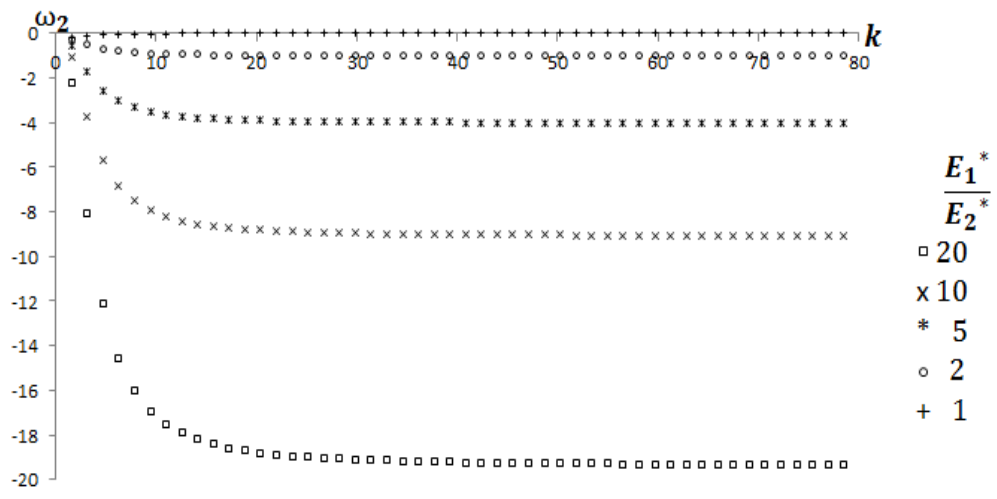

FIGURE 21. Stability results as a function of the relative volatility. The results are shown for $C a=10^{-3}, \bar{r}=0.5$ and $\gamma_{1}^{\star} / \gamma_{2}{ }^{\star}=0.5$.

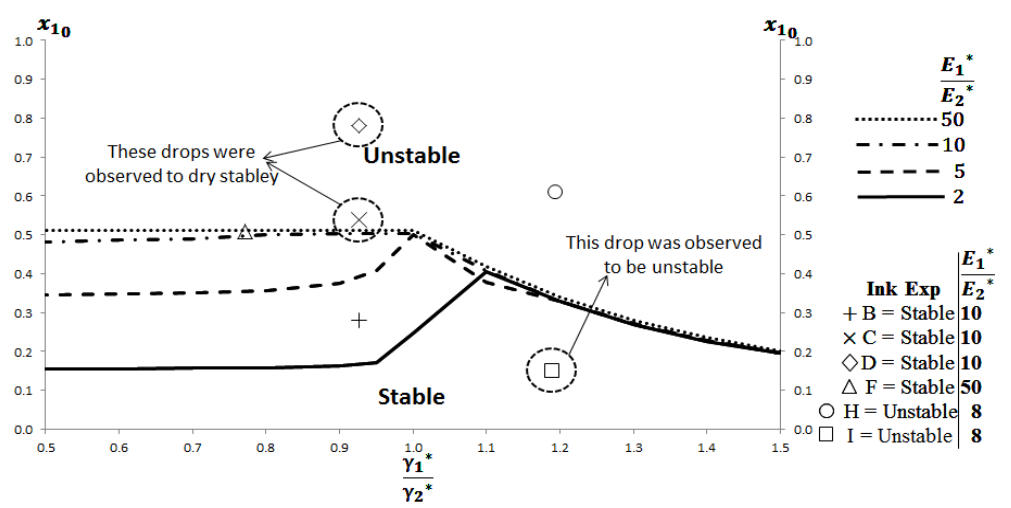

Figure 22. Global stability locus after a time $\bar{t}=10^{-3}$ and for a capillary number $C a=10^{-3}$. The results are shown as a function of the relative volatility, surface tension ratio and the initial mole fraction of liquid 1 and for the first horizontal velocity limit, defined in section 2.3, equation 2.26. The experimental results are superimposed for inks B, C, D, F, H and I. The contradictory evidence is highlighted and enclosed within a dashed circle.

\subsubsection{The global stability locus}

In Figure 22, the global stability locus is presented. The stability of inks B, C, D, F, H and I from the experiments are superimposed for comparison. The global stability locus represents the prediction for the stability of a binary liquid droplet, with the three key properties $\frac{E_{1}{ }^{\star}}{E_{2}{ }^{\star}}, \frac{\gamma_{1}{ }^{\star}}{\gamma_{2}{ }^{\star}}$ and $x_{10}$. It takes into account the spatial dependence of the stability. Only scenarios that are stable at every location within the droplet are marked as stable. The region below each of the loci is the 'stable' region. The region above each of the loci is the 'unstable' region.

It should be noted that the analysis has been performed at one specific moment in time, $\bar{t}=10^{-3}$. Even the stable situations shown could become unstable at a later time, when the height $\bar{h}(\bar{r})$ and composition $x_{1}(\bar{r})$ profiles are different. Consequently, it could be expected that many more situations are unstable. That said, the analysis does not account for diffusion or the presence of polymer, both of these will have a stabilising effect.

- The instability is caused by a surface tension differential along the droplet surface. The volatility difference of the two liquids, sets up a composition gradient within the droplet, with the edge becoming rich in the less volatile liquid. This composition gradient 


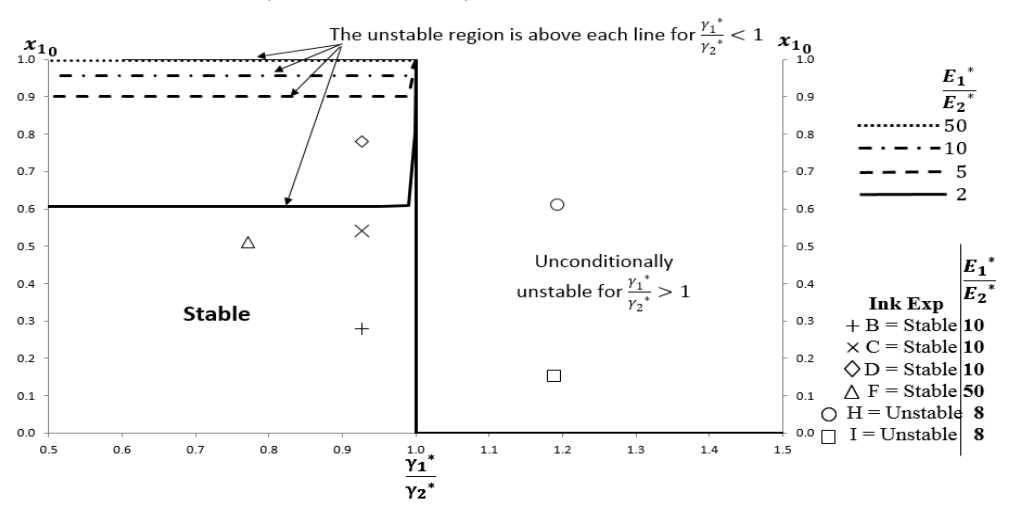

Figure 23. Global stability locus after a time $\bar{t}=10^{-3}$ and for a capillary number $C a=10^{-3}$. The results are shown as a function of the relative volatility, surface tension ratio and the initial mole fraction of liquid 1 and for the second horizontal velocity limit, defined in section 2.3, equation 2.28. The experimental results are superimposed for inks B, C, D, F, H and I.

results in an associated surface tension gradient along the droplet surface. From equation 2.23 , it is clear that the horizontal velocity depends not only on the surface tension at that location but also the surface tension gradient. The large surface tension gradient causes a large velocity and associated rapid change in height. This large height change causes a further change to the composition and so also the surface tension differential. The mechanism accentuates as time proceeds.

- For larger initial mole fractions of more volatile component, $x_{10}$, the $x_{1}(\bar{r})$ profile is sharper, with a large drop to $x_{1}=0$ at the edge. The composition gradient and associated surface tension gradient is larger. Hence, the onset of an instability becomes more likely.

- For larger relative volatilities, the $x_{1}(\bar{r})$ profile is not as sharp (see Figure 4). The less volatile rich region is more diffuse and less confined to the periphery. The composition gradient is reducued and so the onset of an instability is less likely.

Figure 22 suggests that a surface tension ratio less than unity is equally as likely to cause an instability as a surface tension ratio greater than unity. When polymer is introduced, however, there is likely to be some deviation from this behaviour. In section 6.3 .1 , it was noted that an instability is most likely to arise at $\sim \bar{r}=0.99$ for a surface tension ratio less than unity. In a system containing polymer, the growth of a perturbation would be arrested at this location, due to gelation of the polymer. This could explain why inks C \& D, circled in Figure 22, were observed to dry stably. When the surface tension ratio is greater than unity, it was noted in section 6.3.1 that the instability is most likely to arise at $\sim \bar{r}=0.1$. At this location, polymer gelation is unable to arrest the instability and so perturbations will continue to grow. The global stability loci is valid for time $\bar{t}=10^{-3}$. As time proceeds many more situations are likely to become unstable, due to the change in liquid composition gradients. This could explain why Ink I was observed to be unstable.

\subsubsection{Comparison with the second horizontal velocity limit}

The linear stability analysis can also be performed for the second horizontal velocity limit, defined in section 2.3, equation 2.28. The matrix coefficients in equation 6.3 are different and are provided in supplementary information. In Figure 23, the global stability locus is presented. The stability of inks B, C, D, F, H and I from the experiments are superimposed for comparison. 
The system is unconditionally unstable when the surface tension ratio is greater than unity. By contrast, when the surface tension ratio is less than unity, the system is stable, unless the initial mole fraction of the more volatile liquid is very large. There are no inconsistencies between the stability locus shown in Figure 23 and the observations of the experiments for inks B, C, D, F, H \& I.

\section{Proposed mechanism for the propagation of the instability}

Whilst the actual magnitude of the horizontal velocity will be between that of the first and second limits (equations 2.26 and 2.28, in section 2.3), it is fair to say that the experimental results displayed in section 5 , reflect the results for the numerical simulations of the first limit. Therefore, only this limit will be considered from now on. This first horizontal velocity limit corresponds to a zero-shear stress condition on the droplet surface. A Marangoni flow will act to momentarily ensure that the surface composition is spatially uniform. The liquid composition can still vary instantaneously and also in the liquid region below the surface. This will depend on the height at each location within the droplet, the relative volatility of the liquids, the previous liquid composition and the local strength of the horizontal velocity.

The linear stability analysis of section 6 was conducted a time $\bar{t}=10^{-3}$ after the commencement of evaporation. Thus the influence of the liquid composition gradient could be considered. However, it is not possible to use the linear stability analysis to track the evolution of the instability with time. It can only indicate w hether or not an instability will initiate at early times. Once perturbations have grown to an extent that they are no longer very small, it is no longer a good approximation to ignore higher order perturbation terms. The transience of the system is complicated by the evolution of the base state, as well as the perturbation component. Additionally, the perturbations can move laterally, indicated by a complex solution for $\omega$.

Instead we use a flow direction argument to explain the build-up and sharpening of the coffee-ring shape at the edge of the droplet, when the surface tension ratio is greater than unity (see Figures 11 and 16). Flow occurs from regions of high pressure to regions of low pressure. In other words, in the direction that the horizontal pressure gradient is negative.

Differentiating the Young-Laplace equation results in the following equation

$$
\frac{d p}{d r}=-\gamma \frac{d \kappa}{d r}-\kappa \frac{d \gamma}{d r}
$$

Initially the droplet has positive curvature and $\frac{d \kappa}{d r}>0$. Hence, when the surface tension ratio is less than unity, $\frac{d \gamma}{d r}>0$ and so the velocity is outward at all locations. Typically, we do not see an instability in this situation, unless the initial mole fraction of the more volatile liquid is very large.

When the surface tension ratio is greater than unity, $\frac{d \gamma}{d r}<0$. This leads to a competition between the two terms in equation 7.1 for control of the flow direction. To determine which term dominates and at which location, we define the term $\chi$ below

$$
\chi=\frac{\gamma \frac{d \kappa}{d r}}{\kappa \frac{d \gamma}{d r}}
$$

If $\chi>1$ the flow direction will be outwards, if $\chi<1$ the flow direction will be inwards. 
At the edge $\left|\frac{d \gamma}{d r}\right|$ is very large and so $\chi<1$ and the flow is inward. Elsewhere, $\frac{d \kappa}{d r}$ dominates and the flow is outward. This suggests there will be a pile-up of material at the location where the flow direction switches. This behaviour will continue to be exhibited as time proceeds.

Soon after, a coffee-ring shaped surface profile arises. There is a region of negative curvature from the centre, up to the horizontal location of the ring peak. The region outwards from the ring peak, up to the edge of the droplet still has positive curvature. In terms of liquid composition, there are three regions. At the centre and at the edge, the liquid is rich in the less volatile liquid, due to small height. The negative curvature region on the inward edge of the ring will have less depletion of the more volatile liquid. By considering the group $\chi$ at each location, the flow directions can be determined. It is found that the coffee-ring style surface shape sharpens and does not self-correct.

\section{Conclusion}

A model, based on lubrication theory has been used to predict the height, polymer volume fraction and liquid composition evolution with time for thin, binary liquid droplets. The parameters controlling the final film shape have been studied. In addition, a linear stability analysis was conducted, to predict the parameter values that may give rise to an instability, at early times.

The instability occurs due to the surface tension differential across the droplet surface. The instability is most likely to occur when the mole fraction of the more volatile liquid is large, since this gives rise to a sharper gradient in liquid composition, when the liquids have differing volatility. If the surface tension of the more volatile liquid is less than that for the less volatile liquid, the critical position at which an instability would occur is close to the edge. In polymer laden droplets, this instability would be arrested by gelation of the polymer. If the opposite surface tension differential existed, the instability would onset towards the centre of the droplet. The polymer could not stabilise the system at this location. We have consistently observed a rapid change in height and an extreme coffeering formation, in this eventuality, for both the numerical solution and experimental results. A more comprehensive and detailed picture of the instability could be achieved by a stability analysis that accounts for polymer, diffusional effects and temporal variation, but this falls outside the scope of the present study.

Under standard conditions, the liquid-liquid diffusion dominates convection. If it were not for the reduction of liquid-liquid diffusion caused by polymer gelation, we would expect there to be minimal change to the film profile, no matter what relative volatility and mixing ratio were chosen. When the temperature is increased, convection is no longer insignificant. Under these conditions, we have observed, both numerically and experimentally, a reduction in the extent of coffee-ring formation when the relative volatility of the two liquid components is increased. The reason is that the differential evaporation rate initialises a composition differential within the droplet, with a less volatile rich region at the periphery of the droplets and a more volatile central region. If the liquid-liquid diffusion is not completely dominant, the composition gradient is not completely equilibrated. The evaporation rate therefore becomes larger towards the centre of the droplet, when compared to a droplet containing only one liquid. The outward capillary flow driving coffee-ring formation is thus reduced. 


\section{Acknowledgements}

This research has been funded by the Engineering \& Physical Sciences Research Council, UK and CASE studentship funding from Cambridge Display Technology Ltd., UK.

We thank Dr Mark Dowling of Cambridge Display Technology Ltd., for help with the experimental setup.

\section{REFERENCES}

Ahn, B. Y., Duoss, E. B., Motala, M. J., Guo, X., Park, S. I., Xiong, Y., Yoon, J., Nuzzo, R. G., Rogers, J. A. \& Lewis, J. A. 2009 Omnidirectional printing of flexible, stretchable, and spanning silver microelectrodes. Science 323(5921), 1590-1593.

Angenendt, P. 2005 Progress in protein and antibody microarray technology. Drug Discovery Today 10(7), 503-511.

Babatunde, P. O., Hong, W. J., Makaso, K. \& Fukai, J. 2013 Effect of solute and solvent derived marangoni flows on the shape of polymer films formed from drying droplets. AIChE Journal 59(3), 699-702.

Basi, D., Hunsche, M. \& NogA, G. 2013 Effects of surfactants and the kinetic energy of monodroplets on the deposit structure of glyphosate at the micro-scale and their relevance to herbicide bio-efficacy on selected weed species. Weed Research 53(1), 1-11.

Bhardwaj, R., Fang, X., Somasundaran, P. \& Attinger, D. 2010 Self-assembly of colloidal particles from evaporating droplets: Role of dlvo interactions and proposition of a phase diagram. Langmuir 26(11), 7833-7842.

Bright, C. J., Carter, J., Cacheiro, M. \& Lyon, P. 2010 Ink jet deposition; comprises electroluminescent material deposited on substrate dissolved in solvent system comprising first high boiling solvent which exhibits low solubility of material to be deposited, and second low boiling solvent which exhibits high solubility of material to be deposited. priority date feb 27, 2001. filing date oct 9, 2008. Tech. Rep.. Cambridge Display Technology Ltd.

Brutin, D., Sobac, B., Loquet, B. \& Sampol, J. 2011 Pattern formation in drying drops of blood. J. Fluid Mech. .

Chow, E., Herrmann, J., Barton, C. S., Raguse, B. \& Wieczorek, L. 2009 Inkjet-printed gold nanoparticle chemiresistors: Influence of film morpholgy and ionic strength on the detection of organics dissolved in aqueous solution. Analytica Chimica Acta 632, 135-142.

DAhaeck, S., Rednikov, A. \& Colinet, P. 2014 Vapour based interferometric measurement of local evaporation rate and interfacial temperature of evaporating droplets. Langmuir 30(8), 2002-2008.

VAn Dam, D. B. \& Kuerten, J. G. M. 2008 Modelling the drying of inkjet printed structures and experimental verification. Langmuir 24(2), 582-589.

DE Gans, B. J., Duineveld, P. C. \& Schubert, U. S. 2004 Inkjet printing of polymers: state of the art and future developments. Advanced Materials 16(3), 203-213.

DE Gans, B. J. \& Schubert, U. S. 2004 Inkjet printing of well-defined polymer dots and arrays. Langmuir 20(18), 7789-7793.

Deegan, R.D. 2000 Pattern formation in drying drops. Physical Review E 61(1), 475-485.

Deegan, R.D., Bakajin, O., Dupont, T. F., Huber, G., Nagel, S. R. \& Witten, T. A. 1997 Capillary flow as the cause of ring stains from dried liquid drops. Nature 389, 827-829.

Deegan, R.D., Bakajin, O., Dupont, T. F., Huber, G., Nagel, S. R. \& Witten, T. A. 2000 Contact line deposits in an evaporating drop. Physical Review E 62(1), 756-765.

Deng, Y., Zhu, X. Y., Kienlen, T. \& Guo, A. 2006 Transport at the air/water interface is the reason for rings in protein microarrays. Journal of the American Chemical Society 128(9), 2768-2769.

Dijskman, J. F., Duineveld, P. C., Hack, M. J. J., Pierik, A., Rensen, J., Rubingh, J. E., Schram, I. \& Vernhout, M. M. 2007 Precision ink jet printing of polymer light emitting displays. Journal of Materials Chemistry 17, 511-522.

DiJskman, J. F. \& Pierik, A. 2008 Fluid dynamical analysis of the distribution of ink jet printed biomolecules in microarray substrates for genotyping applications. Biomicrofluidics p. 044101. 
Dugyala, V. R. \& Basavaraj, M. G. 2014 Control over coffee-ring formation in evaporating liquid drops containing ellipsoids. Langmuir 30(29), 8680-8686.

EALES, A. D. 2015 P-oled displays, evaporating droplets and coffee-ring formation: an investigation of the parameters controlling the film shape resulting from drying of droplets containing polymer. PhD Thesis, University of Cambridge .

Eales, A. D., Dartnell, N., Goddard, S. \& Routh, A. F. 2015 a Evaporation of pinned droplets containing polymer - an examination of the important groups controlling final shape. AIChE Journal 61(5), 1759-1767.

Eales, A. D., Dartnell, N., Goddard, S. \& Routh, A. F. $2015 b$ The impact of trough geometry on film shape. a theoretical study of droplets containing polymer, for p-oled display applications. Journal of Colloid \& Interface Science 458, 53-61.

Eral, H. B., Mampallil Augustine, D., Duits, M H. G. \& Mugele, F. 2011 Suppressing the coffee stain effect: how to control colloidal self-assembly in evaporating drops using electrowetting. Soft Matter 7, 4954-4958.

Escobedo, J. \& Ali Mansoori, G. 1998 Surface tension prediction for liquid mixtures. AIChE Journal 44(10), 2324-2332.

Fischer, B. J. 2002 Particle convection in an evaporating colloidal droplet. Langmuir 18(1), $60-67$.

Garcia, B., Miranda, M. J., Leal, J. M., Ortega, J. \& Matos, J. S. 1991 Densities and viscosities of mixing for the binary system of methyl benzoate with n-nonane at different temperatures. Thermochimica Acta 186, 285-292.

Gelderblom, H. 2013 Fluid flow in drying droplets. PhD Thesis, University of Twente .

Goldmann, T. \& Gonzalez, J. S. 2000 Dna-printing: utilization of a standard inkjet printer for the transfer of nucleic acids to solid supports. Journal of Biochemical and Biophysical Methods 42, 105-110.

Heim, T., Preuss, S., Gerstmayer, B., Bosio, A. \& Blossey, R. 2005 Deposition from a drop: morphologies of unspecifically bound dna. Journal of Physics: Condensed Matter 17(9), S703-S715.

Hildebrand, J. H. \& Scott, R. L. 1964 In The solubility of non-electrolytes. Sover Publications Inc. New York, USA.

Hu, H. \& Larson, R. G. 2002 Evaporation of a sessile droplet on a substrate. J. Phys. Chem. B 106, 1334-1344.

Hu, H. \& LARson, R. G. $2005 a$ Analysis of the effects of marangoni stressson the microflow in an evaporating sessile droplet. Langmuir 21, 3972-3980.

Hu, H. \& Larson, R. G. $2005 b$ Analysis of the microfluid flow in an evaporating sessile droplet. Langmuir 21, 3963-3971.

Hu, H. \& LARson, R. G. 2006 Marangoni effect reverses coffee-ring depositions. J. Phys. Chem. B 110, 7090-7094.

Kaneda, M., Hyakuta, K., TAKao, Y., Ishizuka, H. \& Fukai, J. 2008 Internal flow in polymer solution droplets depsoited on a lyophobic surface during a receding process. Langmuir 24, 9102-9109.

Karabasheva, S., Baluschev, S. \& Graf, K. 2006 Microstructures on soluble polymer surfaces via drop deposition of solvent mixtures. Applied Physics Letters 89, 031110-1-3.

Kim, D., Jeong, S., PARK, B. \& Moon, J. 2006 Direct writing of silver conductive patterns: Improvement of film morphology and conductance by controlling solvent compositions. Applied Physics Letters 89, 264101-1-3.

Kölpin, N., Wegeneer, M., Teuber, E., Polster, S., Frey, L. \& Roosen, A. 2013 Conceptional design of nano-particulate ito inks for inkjet printing of electron devices. J. Mater Sci. 48(4), 623-1631.

Krieger, I. M. \& Dougherty, T. J. 1959 A mechanism for non-newtonian flow in suspensions of rigid spheres. Transactions of the Society of Rheology 3, 137-152.

LARson, R. G. 2014 Transport and deposition patterns in drying sessile droplets. AIChE Journal 60(5), 1538-1571.

Naqshbandi, M., Canning, J., Gibson, B. C., Nash, M. M. \& Crossley, M. J. 2012 Room temperature self-assembly of mixed nanoparticles into photonic structures. Nature Communications 3, 1188-1-7. 
Ozawa, K., Nishitani, E. \& Doi, M. 2005 Modeling of the drying process of liquid droplet to form thin film. Japanese Journal of Applied Physics 44(6A), 4229-4234.

PARK, J. \& MoOn, J. 2006 Control of colloidal particle deposit patterns within picoliter droplets ejected by ink-jet printing. Langmuir 22, 3506-3513.

Pierik, A., Boamfa, M., van Zelst, M., Clout, D., Stapert, H., Dijskman, F., Broer, D. \& Wimberger-FriedL, R. 2012 Real time quantitative amplification detection on a microarray: towards high multiplex quantitative pcr. Lab Chip 12, 1897.

Routh, A.F. \& Russel, W. B. 1998 Horizontal drying fronts during solvent evaporation from latex films. AIChE Journal 44(9), 2088-2098.

Routh, A. F. 2013 Drying of thin colloidal films. Reports on Progress in Physics 76(4), 046603$1-30$.

Salamanca, J. M., Ciampi, E., Faux, D. A., Glover, P. M., McDonald, P. J., Routh, A. F., Peters, A. C. I. A., Satguru, R. \& Keddie, J. L. 2001 Lateral drying in thick films of waterborne colloidal particles. Langmuir 17, 3202-3207.

Schmidt, R. L., Randall, J. C. \& Clever, H. L. 1966 The surface tension and density of binary hydrocarbon mixtures: benzene-n-hexane andbenzene-n-dodecane. The Journal of Physical Chemistry pp. 3912-3916.

Sefiane, K., David, S. \& Shanahan, M. E. R. 2008 Wetting and evaporation of ninary mixture drops. J. Phys. Chem. B 112, 11317-11323.

Sekitani, T., Noguchi, Y., Zschieschang, U., Klauk, H. \& Someya, T. 2008 Organic transistors manufactured using inkjet technology with subfemtoliter accuracy. PNAS 105(13), 4976-4980.

Sirringhaus, H., Kawase, T., Friend, R. H., Shimoda, T., Inbasekaran, M., Wu, W. \& Woo, E. P. 2000 High-resolution inkjet printing of all-polymer transistor circuits. Science 290(5499), 2123-2126.

Suri, S. K. \& RAmakrishna, V. 1968 Surface tension of some binary liquid mixtures. The Journal of Physical Chemistry 72(9), 3073-3079.

Talbot, E. L., Berson, A. \& Bain, C. D. 2012 Drying and deposition of picolitre droplets of colloidal suspensions in binary solvent mixtures. nip28: 28th international conference on digital printing technologies and digital fabrication quebec city, canada, sep 9-13 2012 . Tech. Rep.. The Society for Imaging Science and Technology.

Tarasevich, Y. Y., Vodolazskaya, I. V. \& Bondarenko, O. P. 2013 Modeling of spatialtemporal distribution of the components in the drying sessile droplet of biological fluid. Colloids \&5 Surfaces A: Physiochemical \& Engineering Aspects 432, 99-103.

Teichler, A., Perelaer, J. \& Schubert, U. S. 2013 Screening of film formation qualities of various solvent systems for $\pi$ conjugated polymers via combinatorial inkjet printing. Macromolecular Chemistry and Physics 214(5), 547-555.

Tekin, E., De Gans, B. J. \& Schubert, U. S. 2004 Inkjet printing of polymers: from single dots to thin film libraries. Journal of Materials Chemistry 14, 2627-2632.

Vermant, J. 2011 Fluid mechanics: When shpae matters. Nature 476, 286-287.

Wohlfarth, C. 2008 Surface tension of the mixture (1) ethanol; (2) methyl benzoate. in supplement to iv/16, landolt-bornstein - group iv physical chemistry. M. D. Lechner ed. , vol. 24, pp. 439-441. Sover Publications Inc. New York, USA.

Wray, A. W., Papageorgiou, D. T., Craster, R. V., Sefiane, K. \& Matar, O. K. 2014 Electrostatic suppression of the coffee-stain effect. Langmuir 30(20), 5849-5858.

Wu, Y., Eliyahu, J., Liu, P. \& Hu, N. X. 2012 Solvent-based inks comprising silver nanoparticles. priority date mar 7, 2011. filing date mar 7, 2011. Tech. Rep.. Xerox Corporation.

Yamaue, T., Jung, Y. \& DoI, M. 2006 The modeling and simulation of dot formation kinetics in the drying process of polymer solution drop. symposium 8: Multiscale simulation approaches for static and dynamic properties of macromolecular materials. freiburg, germany, sep 18-22, 2006. Tech. Rep.. The proceedings of the Third International Conference Multiscale Materials Modeling.

YAng, W., Liu, C., Zhang, Z., Liu, Y. \& Nie, S. 2012 One step synthesis of uniform organic silver ink drawing directly on paper substrates. J. Mater. Chem. 22, 23012-23016.

Yeo, L. Y., Craster, R. V. \& Matar, O. K. 2003 Marangoni instability of a thin liquid film resting on a locally heated horizontal wall. Physical Review E 67, 056315-1-14. 
Yunker, P.J., Still, T., Lohr, M. A. \& Yodh, A. G. 2011 Suppression of the coffee-ring effect by shape-dependent capillary interactions. Nature 476, 308-311. 